\title{
A Sustainable Approach to Azaheterocyclic Acetamides by Decarboxylative Aminoformylation
}

Zhenxing Zhang ${ }^{\dagger}$, Chunqi Jia ${ }^{\dagger}$, Xiangtao Kong ${ }^{\dagger}$, Muzammal Hussain ${ }^{\dagger}$, Zhenyu Liu ${ }^{\dagger}$, Wanqing Liangt, Ling Jiang ${ }^{\S}$, Hao Jiang ${ }^{+, *}$, Junyan Ma ${ }^{*},+, \|, \#$

†College of Chemistry and Chemical Engineering, Anyang Normal University, Anyang 455000, P. R. China

Guangzhou Institutes of Biomedicine and Health, Chinese Academy of Sciences, Guangzhou 510530, P. R. China

${ }^{\S}$ State Key Laboratory of Molecular Reaction Dynamics, Dalian Institute of Chemical Physics, Chinese Academy of Sciences, Dalian 116023, P. R. China

"Department of Chemistry, Clemson University, Clemson, SC, USA

${ }^{\#}$ Center for Optical Materials Science and Engineering Technology, Clemson, SC, USA

*Corresponding Author E-mail: jiang_hao@gibh.ac.cn;

junyanm@g.clemson.edu.

\section{List of Contents}

General information

Computation Method

Compound data

Spectra
S2

S3-S4

S5-S13

S14-S37

37 pages, 0 figures, 1 table 


\section{General information}

Unless otherwise noted, chemicals and solvents were purchased with the highest purity grade available and were used without further purification. Purification of products was conducted by column chromatography on silica gel (200-300 mesh, from Qingdao, China). NMR spectra were measured on a Bruker ARX400 $\left({ }^{1} \mathrm{H}\right.$ at $400 \mathrm{MHz},{ }^{13} \mathrm{C}$ at $101 \mathrm{MHz},{ }^{19} \mathrm{~F}$ at $\left.376 \mathrm{MHz}\right)$ magnetic resonance spectrometer. Chemical shifts $(\delta)$ are reported in ppm using tetramethylsilane as internal standard $(\mathrm{s}=$ singlet, $\mathrm{d}=$ doublet, $\mathrm{t}=$ triplet, $\mathrm{q}=$ quartet, $\mathrm{dd}=$ doublet of doublets, $\mathrm{td}=$ triplet of doublets, $\mathrm{dt}=$ doublet of triplets, $\mathrm{ddd}=$ doublet of doublet of doublets, $\mathrm{m}=$ multiplet), and coupling constants $(J)$ were reported in Hertz $(\mathrm{Hz})$. Infrared spectra were recorded on a Thermal Fisher Nicolet iS50 Fourier transform spectrometer (FT-IR) and were reported in wave numbers $\left(\mathrm{cm}^{-1}\right)$. HRMS data were obtained on a VG ZAB-HS mass spectrometer, Brucker Apex IV FTMS spectrometer.

The starting materials 1 ware synthesized as reported by Liu ${ }^{[1]}[2]$.

[1] T. Wu, X. Mu, G. Liu, Angew. Chem., Int. Ed. 2011, 50, 12578-12581.

[2] H. Zhang, P. Chen, G. Liu, Synlett 2012, 23, 2749-2752. 


\section{Computation Method}

Quantum chemical calculations were performed by using Gaussian 09 program package ${ }^{1}$. All the geometrical structures were optimized at the PBE0 level by using the def-TZVP basis sets ${ }^{2,3}$, including solvation effects via SMD continuum solvation model ${ }^{4}$. The Gibbs free energy of solutes in the liquid phase is defined as:

$$
\mathrm{G}_{\mathrm{liq}}=\mathrm{E}_{\mathrm{sp}}+\mathrm{G}_{\mathrm{ther}}+\Delta \mathrm{G}_{\mathrm{sol}}(\mathrm{S} 1)
$$

where $\mathrm{E}_{\mathrm{sp}}$ is the single point energy at the B2PYLYP-D3(BJ)/def2-TZVP level on the PBE0/defTZVP structures; $\mathrm{G}_{\text {ther }}$ is the thermal contribution to free energy at PBE0/def-TZVP level; $\triangle \mathrm{G}_{\text {sol }}$ is the solvation free energy at M05-2X/6-31G* level on the PBE0/def-TZVP structures, and the M052X/6-31G* method with SMD model is proved to be a good choice to obtain solvation free energy by previous studies ${ }^{5}$. Oxidation potential of a reaction corresponding to normal hydrogen electrode (NHE) is calculated as ${ }^{6}$ :

$$
\mathrm{E}_{\mathrm{ox}}=\mathrm{G}_{\mathrm{liq}, \mathrm{P}}-\mathrm{G}_{\mathrm{liq}, \mathrm{R}}+\Delta \mathrm{G} \text { (NHE) (S2) }
$$

where $\mathrm{G}_{\mathrm{liq}, \mathrm{P}}$ and $\mathrm{G}_{\mathrm{liq}, \mathrm{R}}$ are Gibbs free energies of products and reactants in the liquid phase, respectively; $\Delta \mathrm{G}$ (NHE)is free energy change associated with the NHE half-reaction, which is -4.44 or $-4.13 \mathrm{eV}$ in water or acetone by experiments, respectively ${ }^{7,8}$. The solvation free energy of proton is water or acetone is -1088 and $-1118 \mathrm{~kJ} / \mathrm{mol}$ reported by previous experiments, respectively ${ }^{7,8}$. All the above computations were performed at the standard conditions.

To assess whether the reactions could proceed under the experimental conditions, DFT calculations were conducted to study free energy changes or oxidation potentials of the reactions. Computed details could be found in Supporting Information. The calculated results are shown in Table S1. Considering that the solvent in our experiment is water and acetone mixed solvent, oxidation potentials of reactions $2 \mathrm{a} \rightarrow 4$ and $6 \rightarrow 7$ could be in the range derived from the two pure solvents. Similarly, the solvation free energy of proton varies from solvents, so the free energy change for reaction $7 \rightarrow 3 \mathrm{a}$ is also given in a range. Since the reduction potential of $\mathrm{S}_{2} \mathrm{O}_{8}{ }^{2-} / \mathrm{SO}_{4}{ }^{2-}$ or $\mathrm{Ag}^{2+} / \mathrm{Ag}^{+}$is 2.05 or $1.98 \mathrm{eV}$ in the aqueous phase, even in consideration of possible errors caused by the solvent, the calculated results in Table 1 show that all the reactions could happen under standard conditions. Because solvation free energy is difficult to calculate at other conditions at present, oxidation potentials or free energy changes at experimental conditions of $60^{\circ} \mathrm{C}$ has not been computed. But the elevated reaction temperature could accelerate the reactions and might make the conversion between 5 and 6 more favorable.

Table S1. Calculated oxidation potentials $\left(\mathrm{E}_{\mathrm{ox}}\right)$ or free energy changes $(\Delta \mathrm{G})$ for the reactions.

\begin{tabular}{|c|c|}
\hline & $\mathrm{E}_{\text {ox }}(\mathrm{eV})$ or $\Delta \mathrm{G}(\mathrm{kJ} / \mathrm{mol})$ \\
\hline $2 \mathrm{a}-\mathrm{e}^{-} \rightarrow 4+\mathrm{CO}_{2}$ & $-0.20 \sim 0.11 \mathrm{eV}$ \\
\hline $4+1 \mathrm{a} \rightarrow 5$ & $-46.56 \mathrm{~kJ} / \mathrm{mol}$ \\
\hline
\end{tabular}




\begin{tabular}{|c|c|}
\hline $5 \rightarrow 6$ & $-11.08 \mathrm{~kJ} / \mathrm{mol}$ \\
\hline $6-\mathrm{e}^{-} \rightarrow 7$ & $-0.04 \sim 0.27 \mathrm{eV}$ \\
\hline $7 \rightarrow 3 \mathrm{a}+\mathrm{H}^{+}$ & $-200.09 \sim-170.09 \mathrm{~kJ} / \mathrm{mol}$ \\
\hline
\end{tabular}

1. M. J. Frisch, G. W. Trucks, H. B. Schlegel, G. E. Scuseria, M. A. Robb, J. R. Cheeseman, G. Scalmani, V. Barone, B. Mennucci, G. A. Petersson, H. Nakatsuji, M. Caricato, X. Li, H. P. Hratchian, A. F. Izmaylov, J. Bloino, G. Zheng, J. L. Sonnenberg, M. Hada, M. Ehara, K. Toyota, R. Fukuda, J. Hasegawa, M. Ishida, T. Nakajima, Y. Honda, O. Kitao, H. Nakai, T. Vreven, J. J. A. Montgomery, J. E. Peralta, F. Ogliaro, M. Bearpark, J. J. Heyd, E. Brothers, K. N. Kudin, V. N. Staroverov, T. Keith, R. Kobayashi, J. Normand, K. Raghavachari, A. Rendell, J. C. Burant, S. S. Iyengar, J. Tomasi, M. Cossi, N. Rega, J. M. Millam, M. Klene, J. E. Knox, J. B. Cross, V. Bakken, C. Adamo, J. Jaramillo, R. Gomperts, R. E. Stratmann, O. Yazyev, A. J. Austin, R. Cammi, C. Pomelli, J. W. Ochterski, R. L. Martin, K. Morokuma, V. G. Zakrzewski, G. A. Voth, P. Salvador, J. J. Dannenberg, S. Dapprich, A. D. Daniels, O. Farkas, J. B. Foresman, J. V. Ortiz, J. Cioslowski, and D. J. Fox, Gaussian 09, Revision D.01, Wallingford CT: Gaussian, Inc. 2013.

2. C. Adamo, and V. Barone, J. Chem. Phys. 1999, 110, 6158-6170.

3. A. Schäfer, C. Huber, and R. Ahlrichs, J. Chem. Phys. 1994, 100, 5829-5835.

4. A. V. Marenich, C. J. Cramer, and D. G. Truhlar, J. Phys. Chem. B 2009, 113, 6378-96.

5. J. Ho, A. Klamt, and M. L. Coote, J. Phys. Chem. A 2010, 114, 13442-13444.

6. P. Winget, E. J. Weber, C. J. Cramer, and D. G. Truhlar, Phys. Chem. Chem. Phys. 2000, 2, 12311239.

7. S. Trasatti, Pure Appl. Chem. 1986, 58, 955-966.

8. S. Trasatti, Electrochim. Acta 1987, 32, 843-850. 


\section{Compound data}<smiles>CN1C(=O)C(C)(CC(=O)Nc2ccc(NC(=O)CC3(C)C(=O)N(C)c4ccccc43)cc2)c2ccccc21</smiles>

3

$N, N^{\prime}$-(1,4-phenylene)bis(2-(1,3-dimethyl-2-oxoindolin-3-yl)acetamide).

To a $15 \mathrm{~mL}$ test tube with a stir bar was charged with $0.6 \mathrm{mmol} \mathrm{N}$-methyl-N-phenylmethacrylamide, $0.2 \mathrm{mmol}$ 2,2'-(1,4-phenylenebis(azanediyl))bis(2-oxoacetic acid), $0.02 \mathrm{mmol}_{\mathrm{AgNO}_{3}}$ and $0.6 \mathrm{mmol}$ $\mathrm{K}_{2} \mathrm{~S}_{2} \mathrm{O}_{8}$ followed by $2 \mathrm{~mL}$ acquis acetone ( $\left.\mathrm{v} / \mathrm{v} 1 / 2\right)$. The resulting mixture was reacted at $60^{\circ} \mathrm{C}$. The reaction was monitored by TLC or GC. Upon completion, the reaction mixture was concentrated under reduced pressure to retrieve acetone. The residue was chromatographed through silica gel eluting with PE/EA to give the desired product 3 .

${ }^{1} \mathrm{H}$ NMR (400 MHz, DMSO) $\delta 9.74$ (s, 2H), 7.28 (d, $J=7.3 \mathrm{~Hz}, 2 \mathrm{H}$ ), 7.22 (s, 2H), 7.19 (s, 4H), $7.00-$ $6.90(\mathrm{~m}, 4 \mathrm{H}), 3.12(\mathrm{~s}, 6 \mathrm{H}), 2.95$ (d, $J=15.6 \mathrm{~Hz}, 2 \mathrm{H}), 2.82(\mathrm{~d}, J=15.6 \mathrm{~Hz}, 2 \mathrm{H}), 1.25(\mathrm{~s}, 6 \mathrm{H})$.

${ }^{13} \mathrm{C}$ NMR (101 MHz, DMSO) $\delta$ 179.91, 167.67, 143.94, 134.57, 133.89, 128.01, 122.71, 122.10, $119.78,108.58,45.58,43.54,26.53,24.85$.

HRMS (ESI) calcd for $\mathrm{C} 30 \mathrm{H} 31 \mathrm{~N} 4 \mathrm{O} 4[\mathrm{M}+\mathrm{H}]+511.2345$, found 511.2351 .

General Procedure : To a $15 \mathrm{~mL}$ test tube with a stir bar was charged with $0.2 \mathrm{mmol}$ alkene 1, 0.3

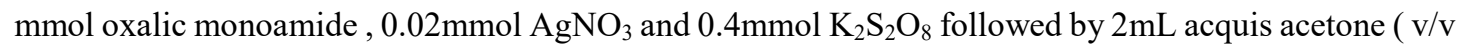
$1 / 2$ ). The resulting mixture was reacted at $60^{\circ} \mathrm{C}$. The reaction was monitored by TLC or GC. Upon completion, the reaction mixture was concentrated under reduced pressure to retrieve acetone. The residue was chromatographed through silica gel eluting with PE/EA to give the desired product 3a-3x. 
<smiles>CN(C)C(=O)CC1(C)C(=O)N(C)C(=O)c2ccccc21</smiles>

$3 a$

2-(2,4-dimethyl-1,3-dioxo-1,2,3,4-tetrahydroisoquinolin-4-yl)-N,N-dimethylacetamide (3a): white solid.

${ }^{1} \mathrm{H}$ NMR (400 MHz, Chloroform- $d$ ) $\delta 8.28(\mathrm{~d}, J=7.8 \mathrm{~Hz}, 1 \mathrm{H}), 7.56(\mathrm{td}, J=7.6,1.5 \mathrm{~Hz}, 1 \mathrm{H}), 7.39(\mathrm{t}, J$ $=7.6 \mathrm{~Hz}, 1 \mathrm{H}), 7.27-7.21(\mathrm{~m}, 1 \mathrm{H}), 3.58(\mathrm{~d}, J=16.6 \mathrm{~Hz}, 1 \mathrm{H}), 3.42(\mathrm{~s}, 3 \mathrm{H}), 3.19(\mathrm{~d}, J=16.7 \mathrm{~Hz}, 1 \mathrm{H})$, $3.02(\mathrm{~s}, 3 \mathrm{H}), 2.73(\mathrm{~s}, 3 \mathrm{H}), 1.52(\mathrm{~s}, 3 \mathrm{H})$.

${ }^{13} \mathrm{C}$ NMR (101 MHz, Chloroform-d) $\delta 177.01,168.98,164.63,144.21,133.49,129.38,126.90$, $125.07,123.34,45.13,43.98,37.08,35.30,30.76,27.26$.

HRMS (ESI) calcd for C15H19N2O3 [M+H]+ 275.1396, found 275.1403.<smiles>Cc1ccc2c(c1)C(CC(=O)N(C)C)(CC(=O)N(C)C)C(=O)N(C)C2=O</smiles>

N,N-dimethyl-2-(2,4,6-trimethyl-1,3-dioxo-1,2,3,4-tetrahydroisoquinolin-4-yl)acetamide $(3 b)$ : white solid.

${ }^{1} \mathrm{H}$ NMR $(400 \mathrm{MHz}$, Chloroform- $d$ ) $\delta 8.16(\mathrm{dd}, J=8.0,1.5 \mathrm{~Hz}, 1 \mathrm{H}), 7.19$ (d, $J=8.1 \mathrm{~Hz}, 1 \mathrm{H}), 7.01$ (s, 1H), 3.56 (d, $J=15.9 \mathrm{~Hz}, 1 \mathrm{H}), 3.40$ (s, 3H), 3.16 (d, $J=16.6 \mathrm{~Hz}, 1 \mathrm{H}), 3.03$ (s, 3H), 2.75 (s, 3H), 2.41 $(\mathrm{s}, 3 \mathrm{H}), 1.50(\mathrm{~s}, 3 \mathrm{H})$.

${ }^{13} \mathrm{C}$ NMR (101 MHz, Chloroform- $d$ ) $\delta 176.25,168.13,163.70,143.30,143.21,128.49,127.10$, $122.93,121.64,44.14,42.99,36.16,34.40,29.97,26.26,21.09$.

HRMS (ESI) calcd for $\mathrm{C} 16 \mathrm{H} 21 \mathrm{~N} 2 \mathrm{O} 3[\mathrm{M}+\mathrm{H}]+289.1552$, found 289.1549.<smiles>Cc1cccc2c1C(=O)N(C)C(=O)C2(C)CC(=O)N(C)C</smiles>

$3 c$

N,N-dimethyl-2-(2,4,8-trimethyl-1,3-dioxo-1,2,3,4-tetrahydroisoquinolin-4-yl)acetamide $\quad(3 \mathbf{c}) \quad$ : white solid. 
${ }^{1} \mathrm{H}$ NMR $(400 \mathrm{MHz}$, Chloroform- $d$ ) $\delta 7.39$ (t, $J=7.7 \mathrm{~Hz}, 1 \mathrm{H}), 7.17(\mathrm{~d}, J=7.6 \mathrm{~Hz}, 1 \mathrm{H}), 7.11(\mathrm{~d}, J=7.8$ $\mathrm{Hz}, 1 \mathrm{H}), 3.58$ (d, $J=16.6 \mathrm{~Hz}, 1 \mathrm{H}), 3.38(\mathrm{~d}, J=1.5 \mathrm{~Hz}, 3 \mathrm{H}), 3.18$ (d, $J=16.5 \mathrm{~Hz}, 1 \mathrm{H}), 3.02(\mathrm{~s}, 3 \mathrm{H})$, $2.79(\mathrm{~s}, 3 \mathrm{H}), 2.74(\mathrm{~s}, 3 \mathrm{H}), 1.50(\mathrm{~s}, 3 \mathrm{H})$.

${ }^{13} \mathrm{C}$ NMR (101 MHz, Chloroform-d) $\delta 176.62,169.11,165.18,145.59,142.84,132.28,130.98$, $123.35,121.45,45.23,43.94,37.05,35.28,31.30,27.21,23.99$.

HRMS (ESI) calcd for $\mathrm{C} 16 \mathrm{H} 21 \mathrm{~N} 2 \mathrm{O} 3[\mathrm{M}+\mathrm{H}]+289.1552$, found 289.1545 .<smiles>COc1ccc2c(c1)C(C)(CC(=O)N(C)C)C(=O)N(C)C2=O</smiles>

2-(6-methoxy-2,4-dimethyl-1,3-dioxo-1,2,3,4-tetrahydroisoquinolin-4-yl)-N,N-dimethylacetamide (3d) : white solid.

${ }^{1} \mathrm{H}$ NMR (400 MHz, Chloroform-d) $\delta 8.23(\mathrm{~d}, J=8.8 \mathrm{~Hz}, 1 \mathrm{H}$ ), 6.90 (dd, $J=8.8,2.4 \mathrm{~Hz}, 1 \mathrm{H}), 6.70$ (d, $J$ $=2.4 \mathrm{~Hz}, 1 \mathrm{H}), 3.86(\mathrm{~s}, 3 \mathrm{H}), 3.56(\mathrm{~d}, J=16.6 \mathrm{~Hz}, 1 \mathrm{H}), 3.38(\mathrm{~s}, 3 \mathrm{H}), 3.11(\mathrm{~d}, J=16.6 \mathrm{~Hz}, 1 \mathrm{H}), 3.02(\mathrm{~s}$, $3 \mathrm{H}), 2.75(\mathrm{~s}, 3 \mathrm{H}), 1.50(\mathrm{~s}, 3 \mathrm{H})$.

${ }^{13} \mathrm{C}$ NMR (101 MHz, Chloroform-d) $\delta 177.01,168.96,164.20,163.72,146.43,131.77,118.28$, $111.55,109.76,55.42,45.31,43.94,37.04,35.29,30.85,27.08$.

HRMS (ESI) calcd for $\mathrm{C} 16 \mathrm{H} 21 \mathrm{~N} 2 \mathrm{O} 4[\mathrm{M}+\mathrm{H}]+305.1501$, found 305.1497 .<smiles>CN(C)C(=O)CC1(C)C(=O)N(C)C(=O)c2ccc(F)cc21</smiles>

$3 e$

2-(6-fluoro-2,4-dimethyl-1,3-dioxo-1,2,3,4-tetrahydroisoquinolin-4-yl)- $N, N$-dimethylacetamide (3e) : white solid.

${ }^{1} \mathrm{H}$ NMR (400 MHz, Chloroform- $d$ ) $\delta 8.29$ (dd, $\left.J=8.7,5.9 \mathrm{~Hz}, 1 \mathrm{H}\right), 7.08$ (td, $\left.J=8.4,2.3 \mathrm{~Hz}, 1 \mathrm{H}\right), 6.91$ (dd, $J=9.6,2.4 \mathrm{~Hz}, 1 \mathrm{H}), 3.60$ (d, $J=16.8 \mathrm{~Hz}, 1 \mathrm{H}), 3.40$ (s, 3H), 3.09 (d, $J=16.8 \mathrm{~Hz}, 1 \mathrm{H}), 3.04$ (s, $3 \mathrm{H}), 2.76$ (s, 3H), 1.51 (s, 3H).

${ }^{19} \mathrm{~F}$ NMR (376 MHz, Chloroform- $d$ ) $\delta-104.48$.

${ }^{13} \mathrm{C}$ NMR (101 MHz, Chloroform-d) $\delta 176.54,168.72,164.82,163.69,147.35,147.27,132.42$, $132.33,121.55,121.52,114.75,114.54,110.57,110.34,45.39,44.02,37.09,35.33,30.63$, 27.28 .

HRMS (ESI) calcd for C15H18FN2O3 [M+H]+ 293.1301, found 293.1291. 
<smiles>CN(C)C(=O)CC1(C)C(=O)N(C)C(=O)c2ccc(Cl)cc21</smiles>

$3 f$

2-(6-chloro-2,4-dimethyl-1,3-dioxo-1,2,3,4-tetrahydroisoquinolin-4-yl)-N,N-dimethylacetamide (3f) : white solid.

${ }^{1} \mathrm{H}$ NMR (400 MHz, Chloroform-d) $\delta 8.20$ (d, $\left.J=8.5 \mathrm{~Hz}, 1 \mathrm{H}\right), 7.39-7.32(\mathrm{~m}, 1 \mathrm{H}), 7.22$ (d, $J=2.0$ Hz, 1H), 3.61 (s, 1H), 3.39 (s, 3H), 3.11 (d, $J=16.8 \mathrm{~Hz}, 1 \mathrm{H}), 3.03$ (s, 3H), 2.74 (s, 3H), 1.51 (s, 3H). ${ }^{13} \mathrm{C}$ NMR (101 MHz, Chloroform- $d$ ) $\delta 176.31,168.71,163.75,146.07,139.88,130.91,127.44$, $123.79,45.22,44.10,37.05,35.29,30.49,27.25$.

HRMS (ESI) calcd for C15H18CIN2O3 [M+H]+ 309.1006, found 309.1007.<smiles>CN(C)C(=O)CC1(CC(=O)N(C)C)C(=O)N(C)C(=O)c2ccc3ccccc3c21</smiles>

2-(1,3-dimethyl-2,4-dioxo-1,2,3,4-tetrahydrobenzo[f]isoquinolin-1-yl)-N,N-dimethylacetamide (3g) : white solid.

${ }^{1} \mathrm{H}$ NMR (400 MHz, Chloroform-d) $\delta 8.40$ (dd, $\left.J=9.3,4.4 \mathrm{~Hz}, 2 \mathrm{H}\right), 7.97-7.90$ (m, 1H), 7.87 (d, $J=$ $8.6 \mathrm{~Hz}, 1 \mathrm{H}), 7.58(\mathrm{dd}, J=6.5,3.3 \mathrm{~Hz}, 2 \mathrm{H}), 4.04(\mathrm{~d}, J=16.6 \mathrm{~Hz}, 1 \mathrm{H}), 3.76(\mathrm{~d}, J=16.6 \mathrm{~Hz}, 1 \mathrm{H}), 3.47$ (s, 3H), 2.90 (s, 3H), 2.61 (s, 3H), 2.00 (s, 3H), 1.57 (s, 3H).

${ }^{13} \mathrm{C}$ NMR (101 MHz, Chloroform- $d$ ) $\delta 178.39,169.35,165.05,140.20,137.06,130.45,128.79$, $128.76,127.26,126.10,125.76,124.77,124.60,46.76,44.02,37.08,35.33,28.36,27.48$. HRMS (ESI) calcd for $\mathrm{C} 19 \mathrm{H} 21 \mathrm{~N} 2 \mathrm{O} 3[\mathrm{M}+\mathrm{H}]+325.1552$, found 325.1544 .<smiles>CN1C(=O)c2ccccc2C(C)(CC(N)=O)C1=O</smiles>

2-(2,4-dimethyl-1,3-dioxo-1,2,3,4-tetrahydroisoquinolin-4-yl)acetamide (3h) : white solid. ${ }^{1} \mathrm{H}$ NMR $\left(400 \mathrm{MHz}\right.$, Chloroform- $d$ and Methanol- $\left.d_{4}\right) \delta 8.13(\mathrm{dd}, J=7.9,1.4 \mathrm{~Hz}, 1 \mathrm{H}), 7.51(\mathrm{td}, J=7.6$, $1.5 \mathrm{~Hz}, 1 \mathrm{H}), 7.33$ (dd, $J=7.7,1.1 \mathrm{~Hz}, 1 \mathrm{H}), 7.29$ (d, $J=8.1 \mathrm{~Hz}, 1 \mathrm{H}), 3.46$ (s, 2H), 3.39 (d, $J=16.2 \mathrm{~Hz}$, $1 \mathrm{H}), 3.30$ (s, 3H), 2.92 (d, $J=16.2 \mathrm{~Hz}, 1 \mathrm{H}), 1.44$ (s, 3H).

${ }^{13} \mathrm{C}$ NMR (101 MHz, Chloroform- $d$ and Methanol- $\left.d_{4}\right) \delta 177.09,172.28,164.83,143.32,133.84$, $128.98,127.30,124.76,124.32,45.39,45.00,30.21,27.17$.

HRMS (ESI) calcd for C13H15N2O3 [M+H]+ 247.1083, found 247.1091. 
<smiles>CN1C(=O)c2ccccc2C(C)(CC(=O)Nc2ccccc2)C1=O</smiles>

2-(2,4-dimethyl-1,3-dioxo-1,2,3,4-tetrahydroisoquinolin-4-yl)-N-phenylacetamide (3i) : white solid.

${ }^{1} \mathrm{H}$ NMR (400 MHz, Chloroform- $d$ and Methanol- $\left.d_{4}\right) \delta 7.92(\mathrm{~d}, J=7.9 \mathrm{~Hz}, 1 \mathrm{H}), 7.34(\mathrm{t}, J=7.6 \mathrm{~Hz}$, $1 \mathrm{H}), 7.19$ (d, $J=7.9 \mathrm{~Hz}, 1 \mathrm{H}), 7.12(\mathrm{t}, J=7.6 \mathrm{~Hz}, 1 \mathrm{H}), 6.95(\mathrm{~d}, J=8.4 \mathrm{~Hz}, 2 \mathrm{H}), 6.88(\mathrm{t}, J=7.7 \mathrm{~Hz}$, 2H), 6.69 (t, $J=7.2 \mathrm{~Hz}, 1 \mathrm{H}), 3.35$ (d, $J=16.2 \mathrm{~Hz}, 1 \mathrm{H}), 3.12(\mathrm{~s}, 3 \mathrm{H}), 3.05$ (s, $1 \mathrm{H}), 2.95$ (d, $J=16.2 \mathrm{~Hz}$, $1 \mathrm{H}), 1.29(\mathrm{~s}, 3 \mathrm{H})$.

${ }^{13} \mathrm{C}$ NMR $\left(101 \mathrm{MHz}\right.$, Chloroform- $d$ and Methanol- $\left.d_{4}\right) \delta 176.98,168.12,143.13,133.61,128.53$, $128.24,126.95,124.36,124.09,123.66,119.62,46.27,44.80,29.68,26.64$.

HRMS (ESI) calcd for C19H19N2O3 [M+H]+323.1396, found 323.1395.<smiles>CN(C)C(=O)CC1(C)C(=O)N(C)c2ccccc21</smiles>

3j

2-(1,3-dimethyl-2-oxoindolin-3-yl)-N,N-dimethylacetamide (3j) : white solid. ${ }^{1} \mathrm{H}$ NMR $\left(400 \mathrm{MHz}, \mathrm{CDCl}_{3}\right) \delta 7.23(\mathrm{~d}, J=7.7 \mathrm{~Hz}, 1 \mathrm{H}), 7.15(\mathrm{~d}, J=7.3 \mathrm{~Hz}, 1 \mathrm{H}), 7.00(\mathrm{t}, J=7.5 \mathrm{~Hz}$, $1 \mathrm{H}), 6.85(\mathrm{~d}, J=7.7 \mathrm{~Hz}, 1 \mathrm{H}), 3.27$ (s, 3H), $3.04-2.90(\mathrm{~m}, 5 \mathrm{H}), 2.76(\mathrm{~s}, 3 \mathrm{H}), 1.36(\mathrm{~s}, 3 \mathrm{H})$. ${ }^{13} \mathrm{C}$ NMR (101 MHz, Chloroform-d) $\delta 180.88,168.88,143.89,134.34,127.64,121.94,121.59$, $108.08,45.74,40.73,37.25,35.34,26.38,24.87$.

HRMS (ESI) calcd for C14H19N2O2 [M+H]+ 247.1447, found 247.1450.<smiles>CCN1C(=O)C(C)(CC(=O)N(C)C)c2ccccc21</smiles>

3k

2-(1-ethyl-3-methyl-2-oxoindolin-3-yl)-N,N-dimethylacetamide (3k) : white solid. ${ }^{1} \mathrm{H}$ NMR $(400 \mathrm{MHz}$, Chloroform- $d$ ) $\delta 7.22(\mathrm{t}, J=7.7 \mathrm{~Hz}, 1 \mathrm{H}), 7.17(\mathrm{~d}, J=7.2 \mathrm{~Hz}, 1 \mathrm{H}), 6.98(\mathrm{t}, J=7.5$ Hz, 1H), $6.86(\mathrm{~d}, J=7.8 \mathrm{~Hz}, 1 \mathrm{H}), 2.95(\mathrm{~s}, 5 \mathrm{H}), 2.76(\mathrm{~s}, 3 \mathrm{H}), 1.36(\mathrm{~s}, 3 \mathrm{H}), 1.29(\mathrm{t}, J=7.2 \mathrm{~Hz}, 3 \mathrm{H})$. ${ }^{13} \mathrm{C}$ NMR (101 MHz, Chloroform-d) $\delta 168.88,142.88,134.58,127.54,121.90,121.74,108.22$, $45.74,40.54,37.27,35.32,34.66,24.88,12.44$.

HRMS (ESI) calcd for $\mathrm{C} 15 \mathrm{H} 21 \mathrm{~N} 2 \mathrm{O} 2[\mathrm{M}+\mathrm{H}]+247.1447$, found 247.1450 . 
<smiles>CC(C)N1C(=O)C(C)(CC(=O)N(C)C)c2ccccc21</smiles>

2-(1-isopropyl-3-methyl-2-oxoindolin-3-yl)-N,N-dimethylacetamide (3I) : white solid. ${ }^{1} \mathrm{H}$ NMR (400 MHz, Chloroform-d) $\delta 7.20$ (d, $\left.J=7.3 \mathrm{~Hz}, 1 \mathrm{H}\right), 7.16$ (d, $\left.J=7.9 \mathrm{~Hz}, 1 \mathrm{H}\right), 7.01$ (d, $J=$ $7.8 \mathrm{~Hz}, 1 \mathrm{H}), 6.96(\mathrm{t}, J=7.5 \mathrm{~Hz}, 1 \mathrm{H}), 4.65(\mathrm{p}, J=7.0 \mathrm{~Hz}, 1 \mathrm{H}), 3.01-2.86(\mathrm{~m}, 5 \mathrm{H}), 2.76(\mathrm{~s}, 3 \mathrm{H}), 1.52$ (dd, $J=7.0,4.6 \mathrm{~Hz}, 6 \mathrm{H}), 1.34$ (s, 3H).

${ }^{13} \mathrm{C}$ NMR (101 MHz, Chloroform-d) $\delta 180.49,168.83,142.45,134.84,127.28,121.93,121.37$, $109.75,45.51,43.61,40.68,37.27,35.30,25.01,19.46,19.03$.

HRMS (ESI) calcd for $\mathrm{C} 16 \mathrm{H} 23 \mathrm{~N} 2 \mathrm{O} 2[\mathrm{M}+\mathrm{H}]+275.1760$, found 275.1771 .<smiles>CN(C)C(=O)CC1(C)C(=O)N(c2ccccc2)c2ccccc21</smiles>

N,N-dimethyl-2-(3-methyl-2-oxo-1-phenylindolin-3-yl)acetamide (3m) : white solid. ${ }^{1} \mathrm{H}$ NMR (400 MHz, Chloroform-d) $\delta 7.51$ (q, $J=4.3,3.4 \mathrm{~Hz}, 4 \mathrm{H}$ ), 7.37 (tt, $J=5.6,2.5 \mathrm{~Hz}, 1 \mathrm{H}$ ), 7.19 $(\mathrm{d}, J=7.3 \mathrm{~Hz}, 1 \mathrm{H}), 7.15(\mathrm{t}, J=7.7 \mathrm{~Hz}, 1 \mathrm{H}), 7.02(\mathrm{t}, J=7.4 \mathrm{~Hz}, 1 \mathrm{H}), 6.79(\mathrm{~d}, J=7.8 \mathrm{~Hz}, 1 \mathrm{H}), 3.11(\mathrm{~d}$, $J=16.5 \mathrm{~Hz}, 1 \mathrm{H}), 3.03(\mathrm{~d}, J=16.5 \mathrm{~Hz}, 1 \mathrm{H}), 2.98$ (s, 3H), 2.78 (s, 3H), $1.48(\mathrm{~s}, 3 \mathrm{H})$.

${ }^{13} \mathrm{C}$ NMR (101 MHz, Chloroform-d) $\delta 168.88,144.13,135.28,134.18,129.41,127.73,127.52$, $127.00,122.34,121.70,109.34,45.86,41.41,37.27,35.38,25.23$.

HRMS (ESI) calcd for C19H21N2O2 [M+H]+ 309.1603, found 309.1601.<smiles>Cc1ccc2c(c1)C(C)(CC(=O)N(C)C)C(=O)N2C</smiles>

30

N,N-dimethyl-2-(1,3,5-trimethyl-2-oxoindolin-3-yl)acetamide (30) : white solid.

${ }^{1} \mathrm{H}$ NMR (400 MHz, Chloroform-d) $\delta 7.03(\mathrm{~d}, J=7.8 \mathrm{~Hz}, 1 \mathrm{H}), 6.97$ (s, 1H), $6.73(\mathrm{~d}, J=7.8 \mathrm{~Hz}, 1 \mathrm{H})$, 3.24 (s, 3H), 2.96 (s, 5H), 2.78 (s, 3H), 2.31 (s, 3H), 1.35 (s, 3H).

${ }^{13} \mathrm{C}$ NMR (101 MHz, Chloroform-d) $\delta 168.98,141.48,134.36,131.31,127.88,122.60,107.78$, $45.79,40.68,37.25,35.36,26.41,24.96,21.15$.

HRMS (ESI) calcd for $\mathrm{C} 15 \mathrm{H} 21 \mathrm{~N} 2 \mathrm{O} 2[\mathrm{M}+\mathrm{H}]+247.1447$, found 247.1447 . 
<smiles>CCCCc1ccc2c(c1)C(C)(CC(=O)N(C)C)C(=O)N2C</smiles>

2-(5-butyl-1,3-dimethyl-2-oxoindolin-3-yl)-N,N-dimethylacetamide (3p)

${ }^{1} \mathrm{H}$ NMR (400 MHz, Chloroform-d) $\delta 7.04(\mathrm{~d}, J=7.9 \mathrm{~Hz}, 1 \mathrm{H}), 6.98(\mathrm{~s}, 1 \mathrm{H}), 6.75(\mathrm{~d}, J=7.8 \mathrm{~Hz}, 1 \mathrm{H})$, $3.24(\mathrm{~s}, 3 \mathrm{H}), 2.94(\mathrm{~d}, J=2.6 \mathrm{~Hz}, 5 \mathrm{H}), 2.77$ (s, 3H), 2.57 (t, $J=7.8 \mathrm{~Hz}, 2 \mathrm{H}), 1.62-1.51(\mathrm{~m}, 2 \mathrm{H}), 1.35$ (d, $J=12.5 \mathrm{~Hz}, 5 \mathrm{H}), 0.92(\mathrm{t}, J=7.3 \mathrm{~Hz}, 3 \mathrm{H})$.

${ }^{13} \mathrm{C}$ NMR (101 MHz, Chloroform- $d$ ) $\delta 180.83,168.97,141.61,136.60,134.18,127.27,121.99$, $107.73,45.85,40.58,37.30,35.43,35.34,33.95,26.39,24.80,22.34,13.91$.

HRMS (ESI) calcd for $\mathrm{C} 18 \mathrm{H} 27 \mathrm{~N} 2 \mathrm{O} 2[\mathrm{M}+\mathrm{H}]+303.2073$, found 303.2078 .<smiles>CN(C)C(=O)CC1(C)C(=O)N(C)c2ccc(C(F)(F)F)cc21</smiles>

$3 q$

2-(1,3-dimethyl-2-oxo-5-(trifluoromethyl)indolin-3-yl)-N,N-dimethylacetamide (3q) : white solid. ${ }^{1} \mathrm{H}$ NMR (400 MHz, Chloroform-d) $\delta 7.52(\mathrm{~d}, J=8.2 \mathrm{~Hz}, 1 \mathrm{H}), 7.34$ (d, $\left.J=1.8 \mathrm{~Hz}, 1 \mathrm{H}\right), 6.91$ (d, $J=$ $8.1 \mathrm{~Hz}, 1 \mathrm{H}), 3.29$ (s, 3H), 3.08 (d, $J=16.8 \mathrm{~Hz}, 1 \mathrm{H}), 3.01$ (s, 1H), 2.98 (s, 3H), 2.75 (s, 3H), 1.36 (s, 3H). ${ }^{19} \mathrm{~F}$ NMR (376 MHz, Chloroform- $d$ ) $\delta-61.19$.

${ }^{13} \mathrm{C}$ NMR (101 MHz, Chloroform- $d$ ) $\delta 180.83,168.49,147.03,135.05,125.50$ (q, $\left.J=4.1 \mathrm{~Hz}\right)$, $124.63(\mathrm{q}, J=271.4 \mathrm{~Hz}), 124.00(\mathrm{q}, J=32.4 \mathrm{~Hz}), 118.30(\mathrm{q}, J=3.7 \mathrm{~Hz}), \quad 107.71,45.63,40.98$, $37.16,35.30,26.54,24.86$.

HRMS (ESI) calcd for C15H18F3N2O2 [M+H]+ 315.1320, found 315.1327.<smiles>CN(C)C(=O)CC1(C)C(=O)N(C)c2ccc(F)cc21</smiles>

2-(5-fluoro-1,3-dimethyl-2-oxoindolin-3-yl)-N,N-dimethylacetamide (3r) : white solid.

${ }^{1} \mathrm{H}$ NMR (400 MHz, Chloroform- $d$ ) $\delta 6.92(\mathrm{t}, J=8.5 \mathrm{~Hz}, 2 \mathrm{H}), 6.75(\mathrm{dd}, J=8.2,4.2 \mathrm{~Hz}, 1 \mathrm{H}), 3.25(\mathrm{~s}$, 3H), 3.02 (d, $J=16.7 \mathrm{~Hz}, 1 \mathrm{H}), 2.97$ (s, 3H), 2.91 (d, $J=16.7 \mathrm{~Hz}, 1 \mathrm{H}), 2.77$ (s, 3H), 1.35 (s, 3H).

${ }^{9}$ F NMR (376 MHz, Chloroform- $d$ ) $\delta-121.68$.

${ }^{13} \mathrm{C}$ NMR (101 MHz, Chloroform-d) $\delta 180.56,168.64,159.08$ (d, $\left.J=239.4 \mathrm{~Hz}\right), 139.83$ (d, $J=1.9$

$\mathrm{Hz}), 136.10(\mathrm{~d}, J=8.0 \mathrm{~Hz}), 113.54(\mathrm{~d}, J=23.2 \mathrm{~Hz}), 109.96(\mathrm{~d}, J=24.8 \mathrm{~Hz}), 108.34(\mathrm{~d}, J=8.2 \mathrm{~Hz})$, $46.18(\mathrm{~d}, J=1.9 \mathrm{~Hz}), 40.75,37.21,35.35,26.52,24.83$.

HRMS (ESI) calcd for C14H17FN2O2 [M+H]+264.1274, found 264.1268. 
<smiles>CN(C)C(=O)CC1(C)C(=O)N(C)c2ccc(Cl)cc21</smiles>

3s

2-(5-chloro-1,3-dimethyl-2-oxoindolin-3-yl)-N,N-dimethylacetamide (3s) : white solid.

${ }^{1} \mathrm{H}$ NMR $(400 \mathrm{MHz}$, Chloroform- $d$ ) $\delta 7.20(\mathrm{dd}, J=8.2,2.1 \mathrm{~Hz}, 1 \mathrm{H}), 7.10(\mathrm{~d}, J=2.0 \mathrm{~Hz}, 1 \mathrm{H}), 6.76(\mathrm{~d}, J$ $=8.3 \mathrm{~Hz}, 1 \mathrm{H}), 3.25(\mathrm{~s}, 3 \mathrm{H}), 3.03(\mathrm{~d}, J=16.7 \mathrm{~Hz}, 1 \mathrm{H}), 2.97(\mathrm{~s}, 3 \mathrm{H}), 2.92(\mathrm{~d}, J=16.7 \mathrm{~Hz}, 1 \mathrm{H}), 2.77(\mathrm{~s}$, $3 \mathrm{H}), 1.34$ (s, 3H).

${ }^{13} \mathrm{C}$ NMR (101 MHz, Chloroform-d) $\delta 180.42,168.56,142.58,136.20,127.46,127.21,122.10$, $108.93,45.92,40.88,37.19,35.35,26.50,24.89$.

HRMS (ESI) calcd for C14H18ClN2O2 [M+H]+ 281.1057, found 281.1068 .<smiles>CN(C)C(=O)CC1(C)C(=O)N(C)c2ccc(Br)cc21</smiles>

2-(5-bromo-1,3-dimethyl-2-oxoindolin-3-yl)-N,N-dimethylacetamide (3t) : white solid.

${ }^{1} \mathrm{H}$ NMR (400 MHz, Chloroform- $d$ ) $\delta 7.35$ (dd, $\left.J=8.2,2.0 \mathrm{~Hz}, 1 \mathrm{H}\right), 7.23(\mathrm{~d}, J=1.9 \mathrm{~Hz}, 1 \mathrm{H}), 6.72(\mathrm{~d}, J$ $=8.2 \mathrm{~Hz}, 1 \mathrm{H}), 3.24(\mathrm{~s}, 3 \mathrm{H}), 3.03(\mathrm{~d}, J=16.8 \mathrm{~Hz}, 1 \mathrm{H}), 2.97(\mathrm{~s}, 3 \mathrm{H}), 2.91(\mathrm{~d}, J=16.8 \mathrm{~Hz}, 1 \mathrm{H}), 2.77(\mathrm{~s}$, $3 \mathrm{H}), 1.34$ (s, 3H).

${ }^{13} \mathrm{C}$ NMR (101 MHz, Chloroform- $d$ ) $\delta 180.32,168.53,143.08,136.59,130.38,124.78,114.57$, $109.49,45.87,40.92,37.19,35.36,26.48,24.92$.

HRMS (ESI) calcd for $\mathrm{C} 14 \mathrm{H} 18 \mathrm{BrN} 2 \mathrm{O} 2[\mathrm{M}+\mathrm{H}]+325.0552$, found 325.0555 .<smiles>CN(C)C(=O)CC1(C)C(=O)N(C)c2ccc(I)cc21</smiles>

2-(5-iodo-1,3-dimethyl-2-oxoindolin-3-yl)-N,N-dimethylacetamide (3u) : white solid. ${ }^{1} \mathrm{H}$ NMR $(400 \mathrm{MHz}$, Chloroform- $d$ ) $\delta 7.55(\mathrm{dd}, J=8.2,1.8 \mathrm{~Hz}, 1 \mathrm{H}), 7.38(\mathrm{~d}, J=1.7 \mathrm{~Hz}, 1 \mathrm{H}), 6.64(\mathrm{~d}, J$ $=8.1 \mathrm{~Hz}, 1 \mathrm{H}), 3.24(\mathrm{~s}, 3 \mathrm{H}), 3.03(\mathrm{~d}, J=16.8 \mathrm{~Hz}, 1 \mathrm{H}), 2.97(\mathrm{~s}, 3 \mathrm{H}), 2.91(\mathrm{~d}, J=16.8 \mathrm{~Hz}, 1 \mathrm{H}), 2.77(\mathrm{~s}$, $3 \mathrm{H}), 1.33(\mathrm{~s}, 3 \mathrm{H})$.

${ }^{13} \mathrm{C}$ NMR (101 MHz, Chloroform-d) $\delta 180.18,168.54,143.77,136.97,136.40,130.22,110.18$, $84.50,45.69,40.96,37.21,35.38,26.44,24.98$.

HRMS (ESI) calcd for C14H18IN2O2 [M+H]+ 373.0413, found 373.0414. 
<smiles>CCOC(=O)c1ccc2c(c1)C(C)(CC(=O)N(C)C)C(=O)N2C</smiles>

ethyl 3-(2-(dimethylamino)-2-oxoethyl)-1,3-dimethyl-2-oxoindoline-5-carboxylate (3v) : white solid.

${ }^{1} \mathrm{H}$ NMR (400 MHz, Chloroform-d) $\delta 8.00(\mathrm{~d}, J=8.2 \mathrm{~Hz}, 1 \mathrm{H}), 7.77(\mathrm{~s}, 1 \mathrm{H}), 6.87(\mathrm{~d}, J=8.2 \mathrm{~Hz}, 1 \mathrm{H})$, 4.35 (q, $J=7.0 \mathrm{~Hz}, 2 \mathrm{H}), 3.09$ (d, $J=16.7 \mathrm{~Hz}, 1 \mathrm{H}), 3.02$ (d, $J=16.8 \mathrm{~Hz}, 1 \mathrm{H}), 2.98$ (s, 3H), 2.73 (s, 3H), 1.39 (t, $J=7.1 \mathrm{~Hz}, 3 \mathrm{H}), 1.35$ (s, 3H).

${ }^{13} \mathrm{C}$ NMR (101 MHz, Chloroform-d) $\delta 181.22,168.57,166.73,148.28,134.45,130.44,124.07$, $122.39,107.47,60.68,45.42,41.12,37.16,35.30,26.58,25.04,14.39$.

HRMS (ESI) calcd for C17H23F3N2O4 [M+H]+ 319.1658, found 319.1649 .<smiles>CN1C(=O)C(C)(CC(N)=O)c2ccccc21</smiles>

$3 w$

2-(1,3-dimethyl-2-oxoindolin-3-yl)acetamide (3w) : white solid.

${ }^{1} \mathrm{H}$ NMR (400 MHz, Chloroform-d) $\delta 7.27$ (d, $\left.J=7.7 \mathrm{~Hz}, 2 \mathrm{H}\right), 7.08(\mathrm{t}, J=7.5 \mathrm{~Hz}, 1 \mathrm{H}), 6.86(\mathrm{~d}, J=7.7$ $\mathrm{Hz}, 1 \mathrm{H}), 6.39(\mathrm{~s}, 1 \mathrm{H}), 5.27(\mathrm{~s}, 1 \mathrm{H}), 3.24(\mathrm{~s}, 3 \mathrm{H}), 2.81(\mathrm{~d}, J=14.9 \mathrm{~Hz}, 1 \mathrm{H}), 2.68(\mathrm{~d}, J=14.9 \mathrm{~Hz}, 1 \mathrm{H})$, $1.45(\mathrm{~s}, 3 \mathrm{H})$.

${ }^{13} \mathrm{C}$ NMR (101 MHz, Chloroform-d) $\delta 180.56,142.79,133.28,128.24,122.92,122.72,108.34$, $45.96,43.45,26.39,23.44$.

HRMS (ESI) calcd for C12H15N2O2 [M+H]+ 219.1134, found 219.1141.<smiles>CN1C(=O)C(C)(CC(=O)Nc2ccccc2)c2ccccc21</smiles>

2-(1,3-dimethyl-2-oxoindolin-3-yl)-N-phenylacetamide (3x) : white solid.

${ }^{13} \mathrm{C}$ NMR (101 MHz, Chloroform-d) $\delta 180.98,167.71,142.75,138.07,133.39,128.85,128.25$, $124.07,122.99,122.68,120.04,108.51,46.12,44.60,26.49,23.64$.

${ }^{1} \mathrm{H}$ NMR (400 MHz, Chloroform-d) $\delta 9.02(\mathrm{~s}, 1 \mathrm{H}), 7.54-7.40(\mathrm{~m}, 2 \mathrm{H}), 7.33-7.18$ (m, 4H), 7.14 $7.00(\mathrm{~m}, 2 \mathrm{H}), 6.85(\mathrm{~d}, J=7.8 \mathrm{~Hz}, 1 \mathrm{H}), 3.24(\mathrm{~s}, 3 \mathrm{H}), 2.94$ (d, $J=15.2 \mathrm{~Hz}, 1 \mathrm{H}), 2.87$ (d, $J=15.3 \mathrm{~Hz}$, $1 \mathrm{H}), 1.45(\mathrm{~s}, 3 \mathrm{H})$.

HRMS (ESI) calcd for C18H19N2O2 [M+H]+ 295.1447, found 295.1450. 
${ }^{1} \mathrm{H}$ NMR, ${ }^{13} \mathrm{C}$ NMR Spectra
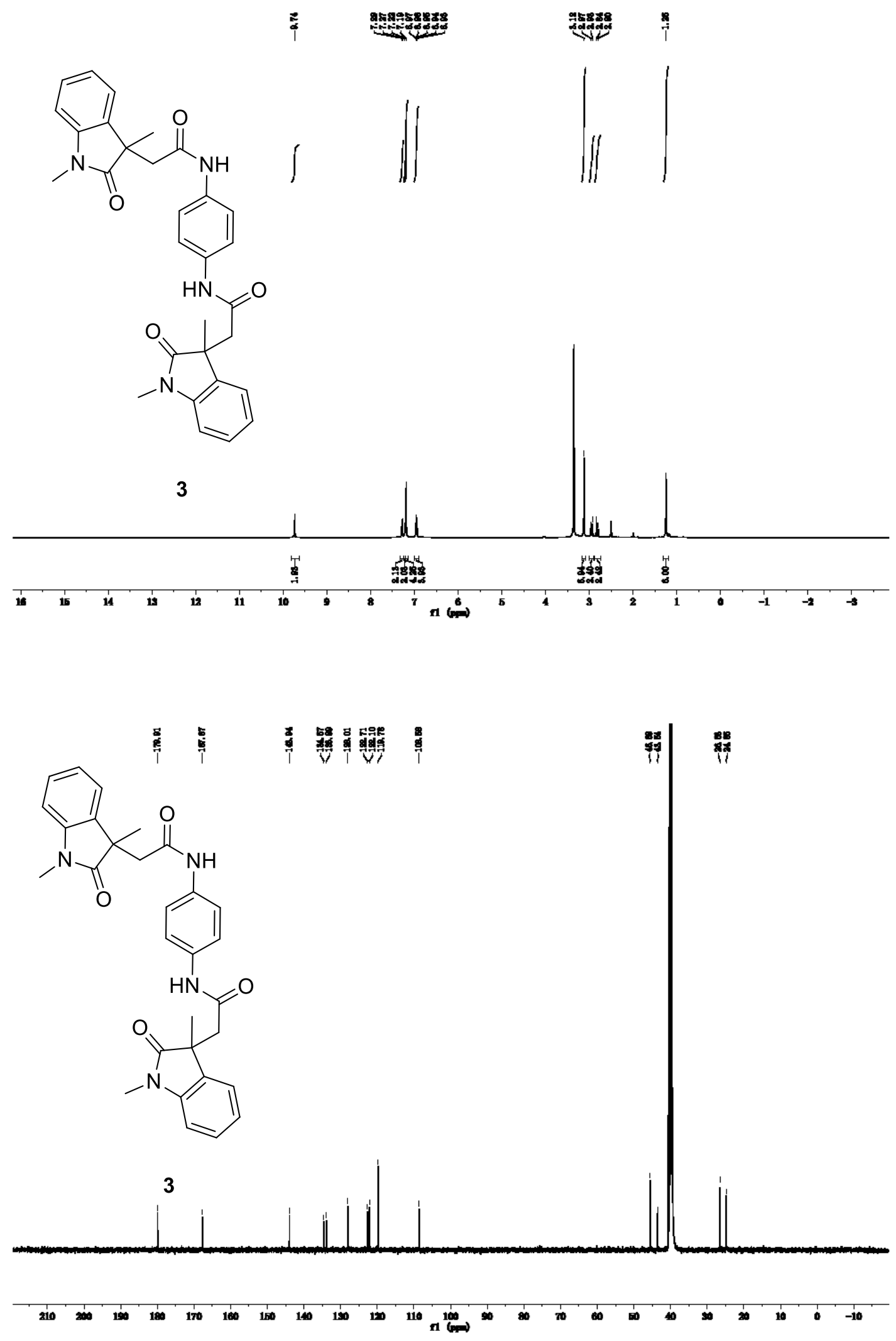


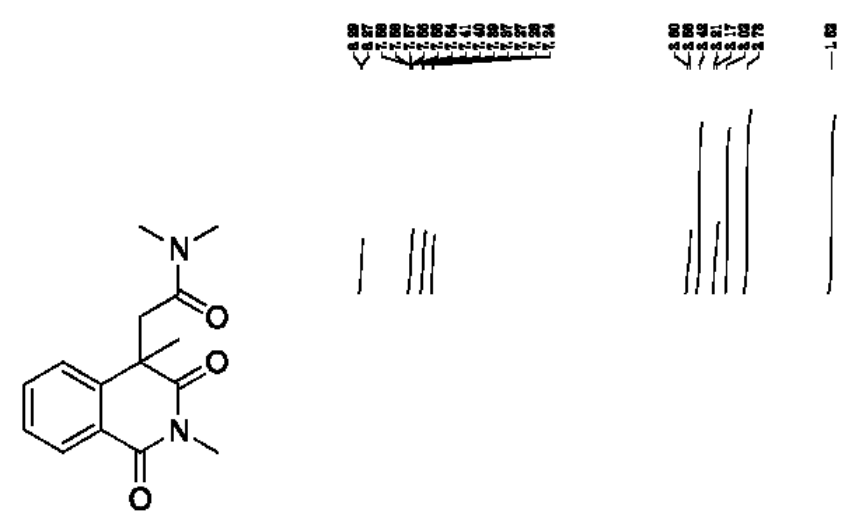

$3 a$

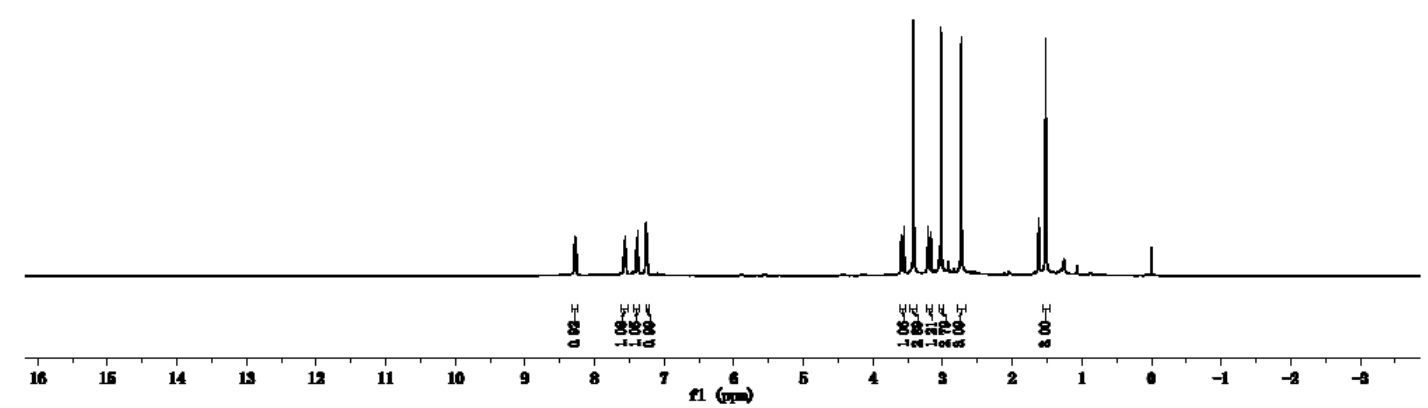

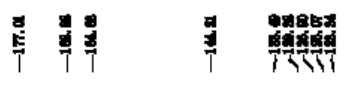

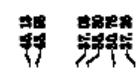

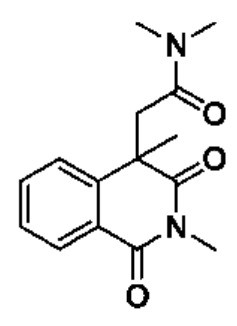

$3 \mathbf{a}$

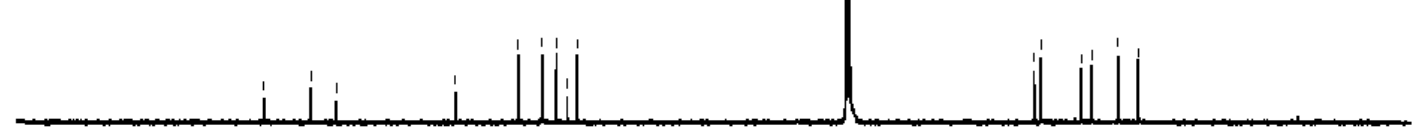

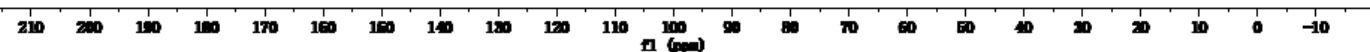




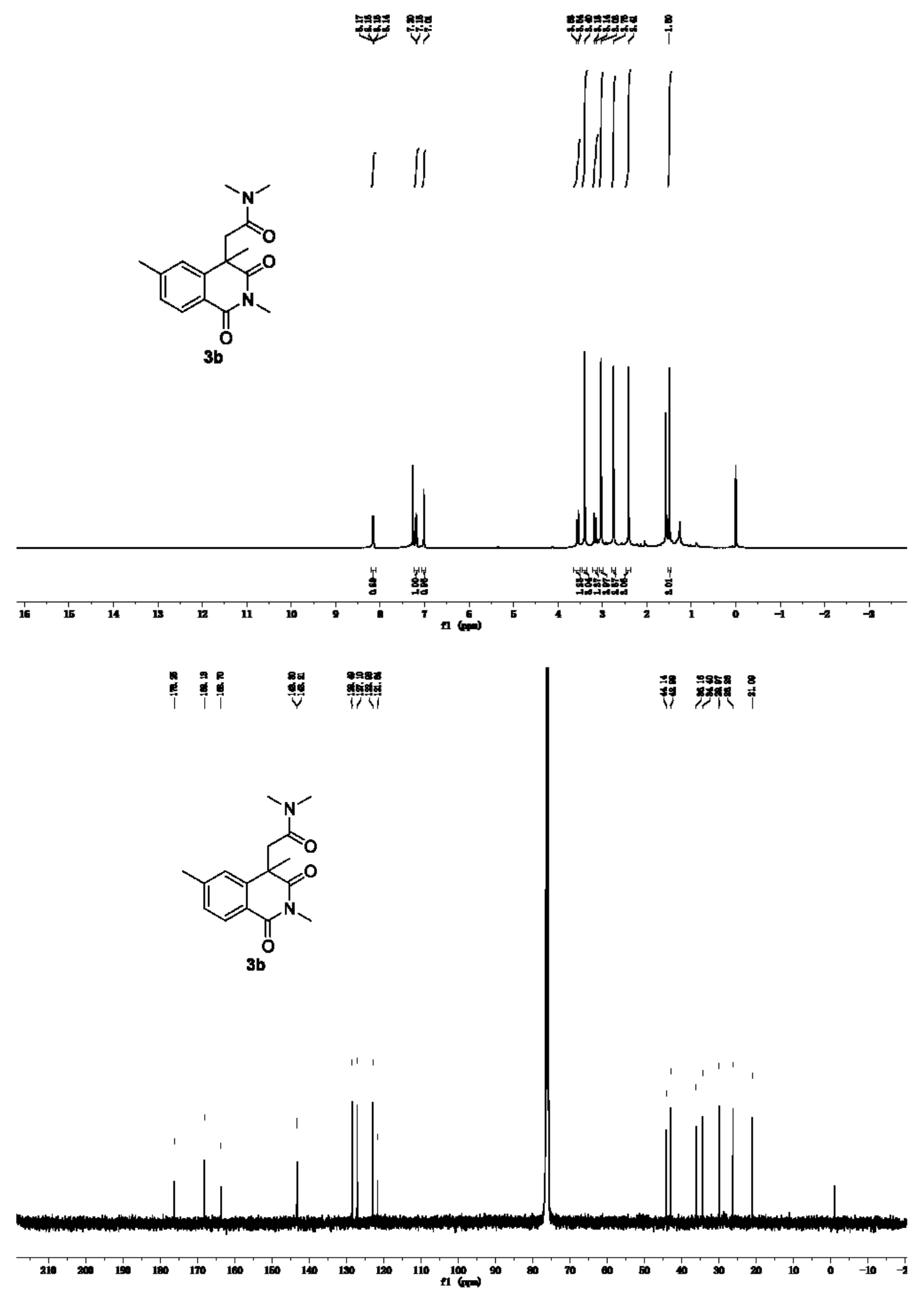




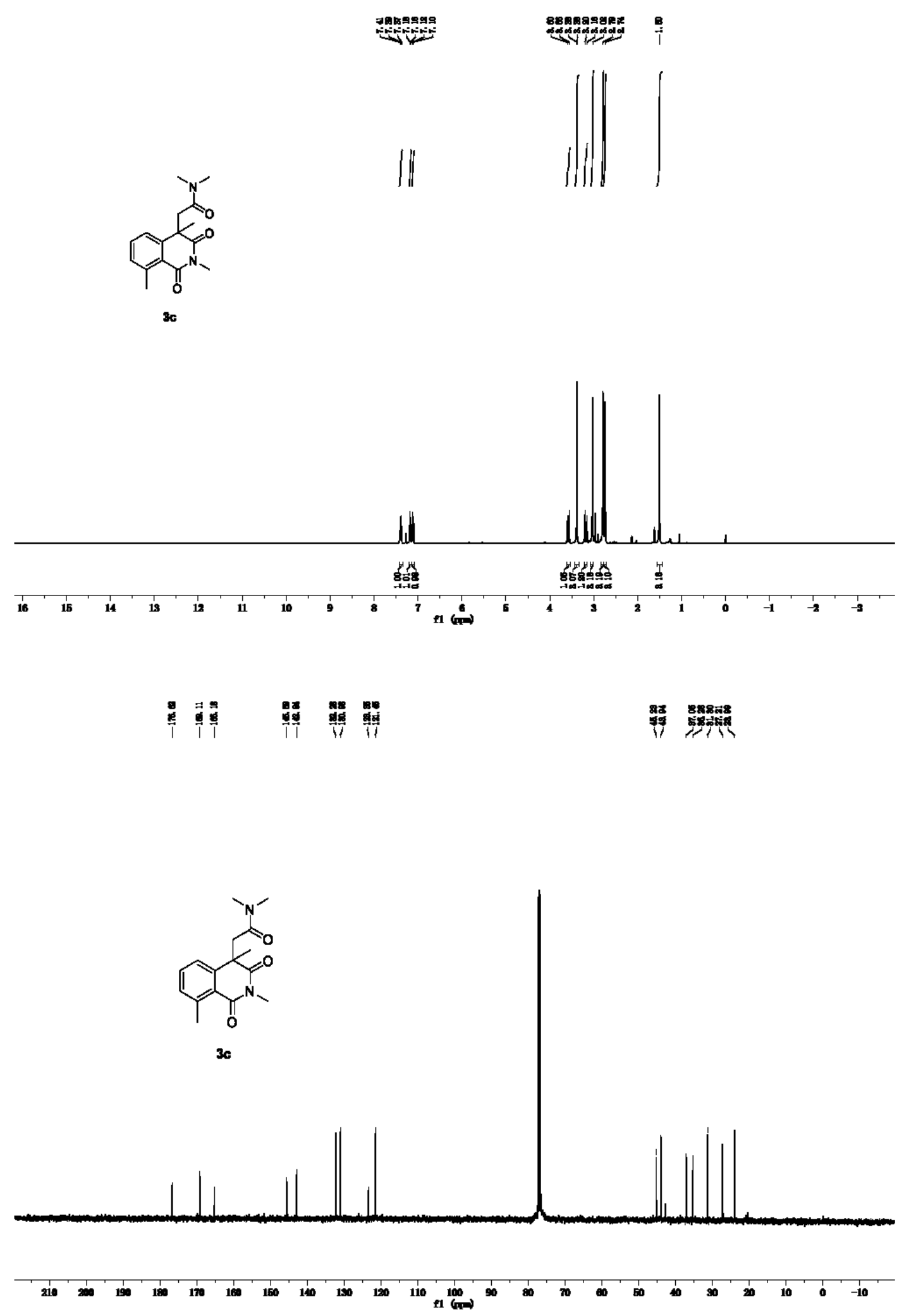




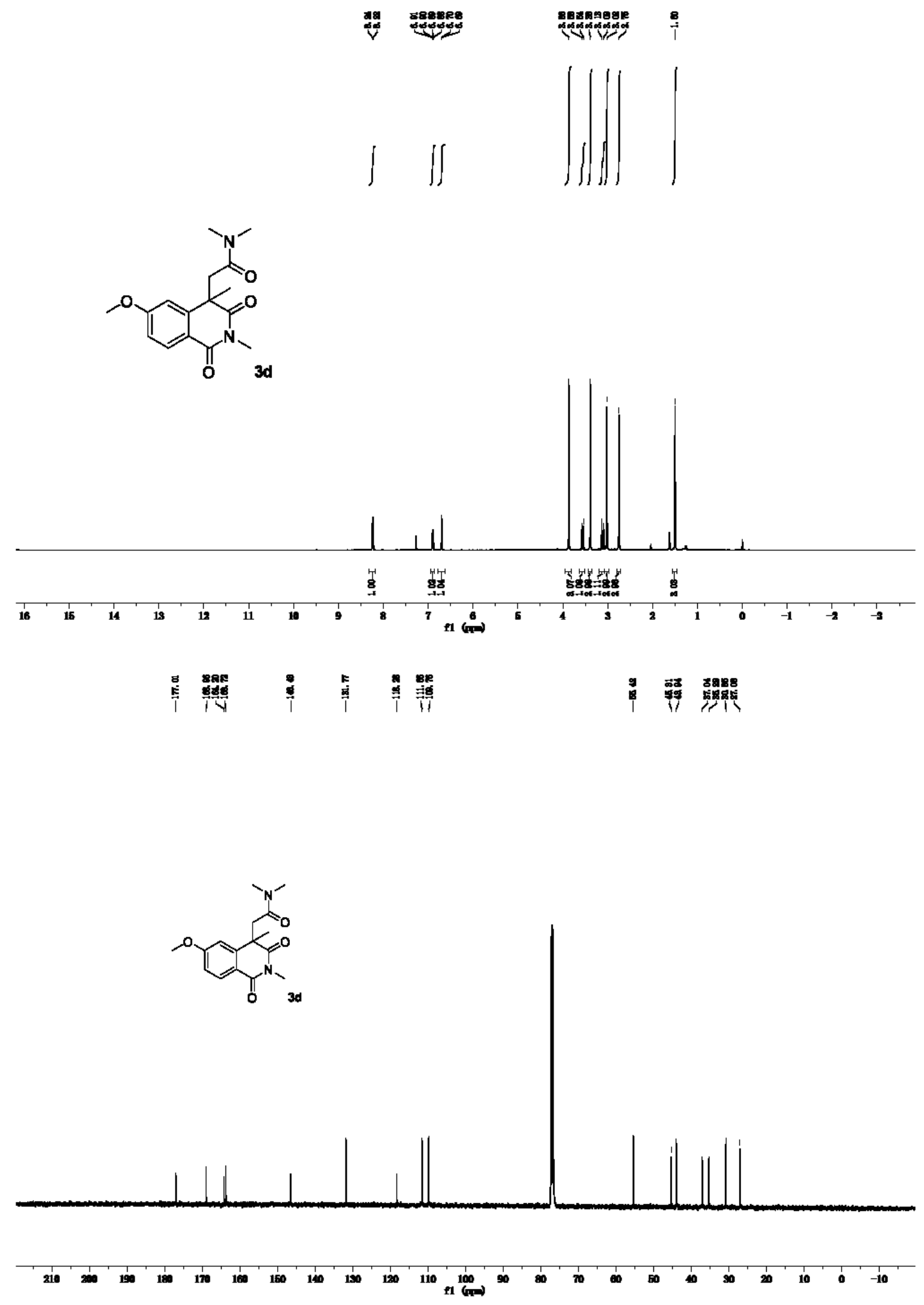


\|\|\|\|

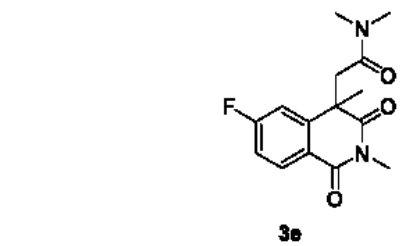

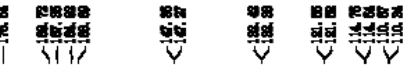

羿8 8988

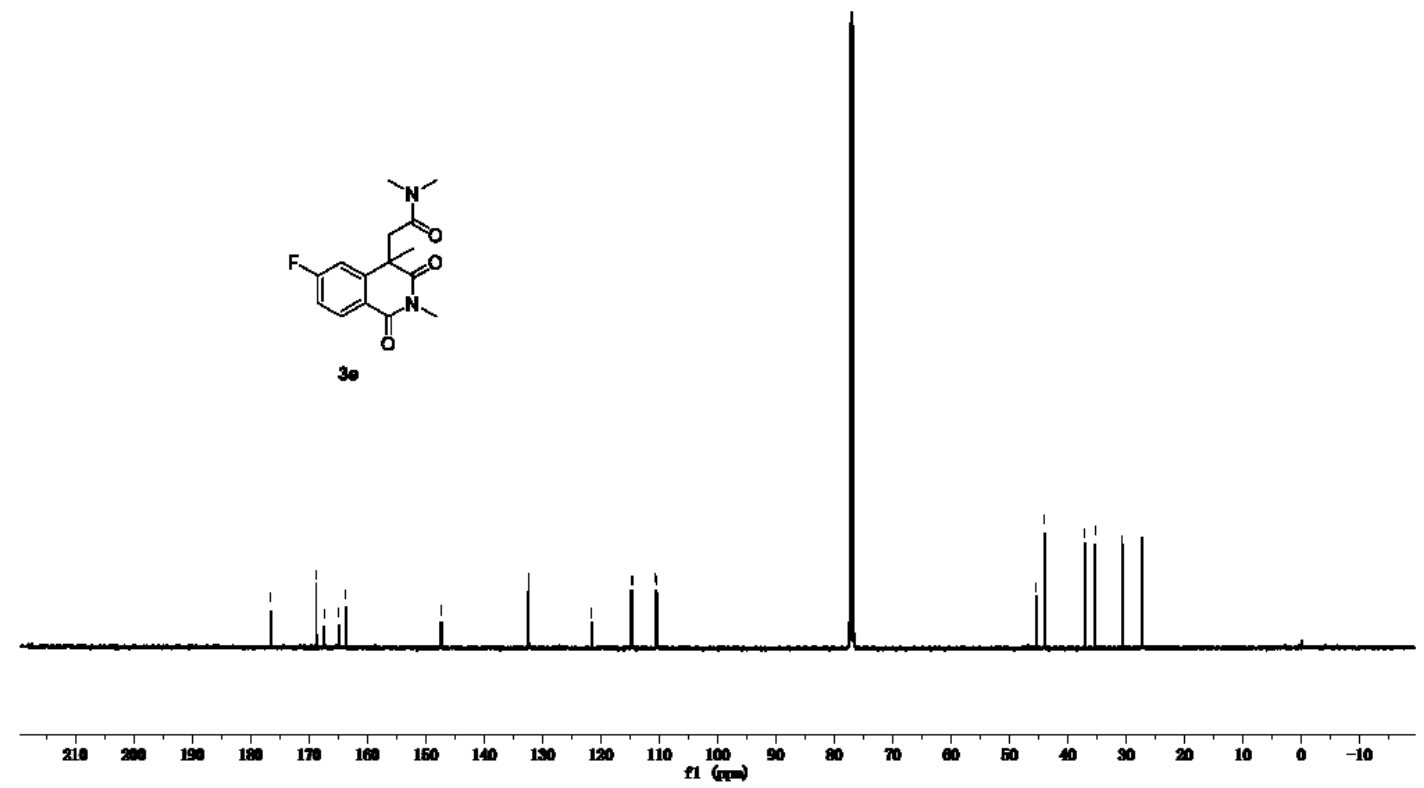




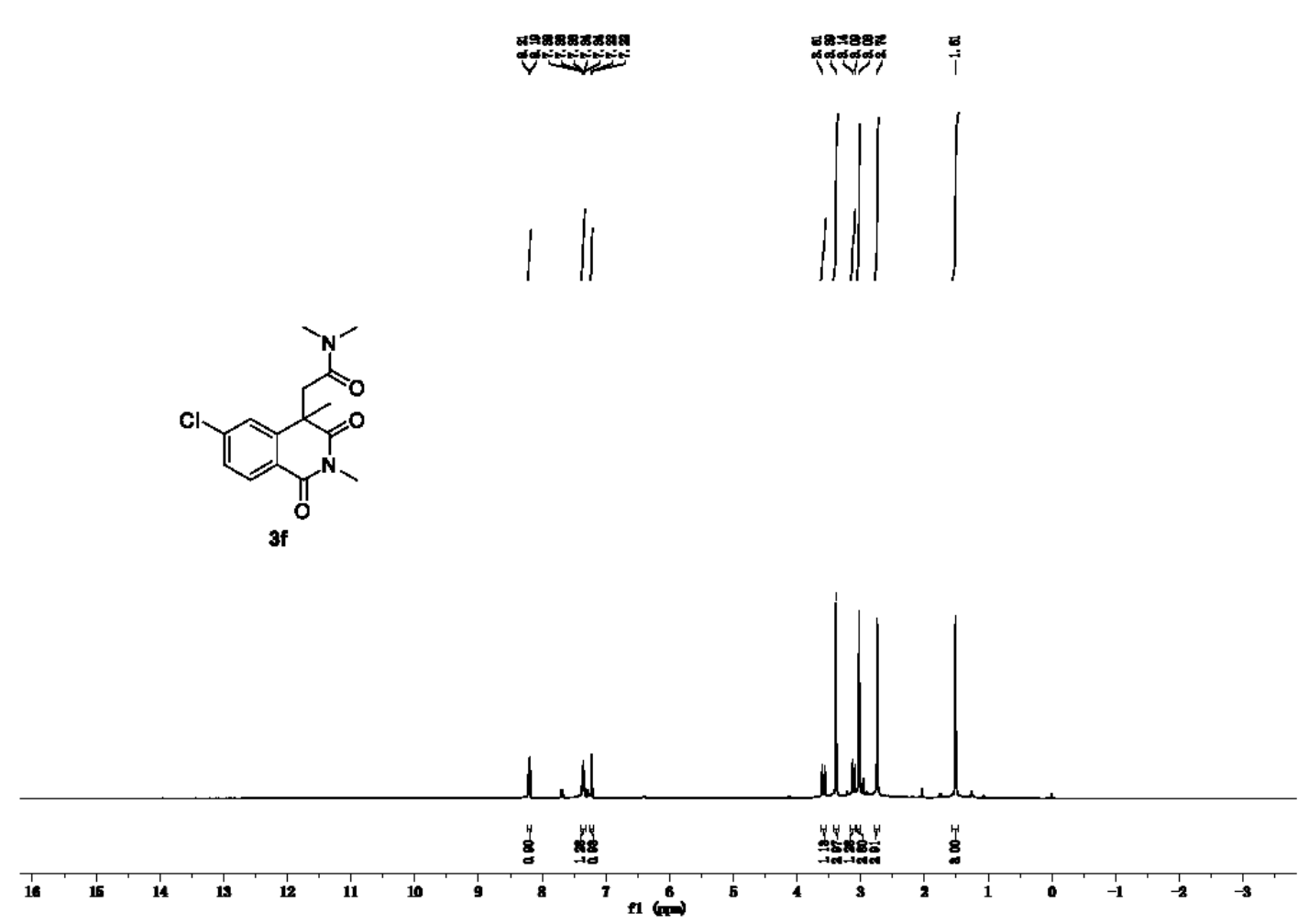

界累

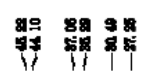
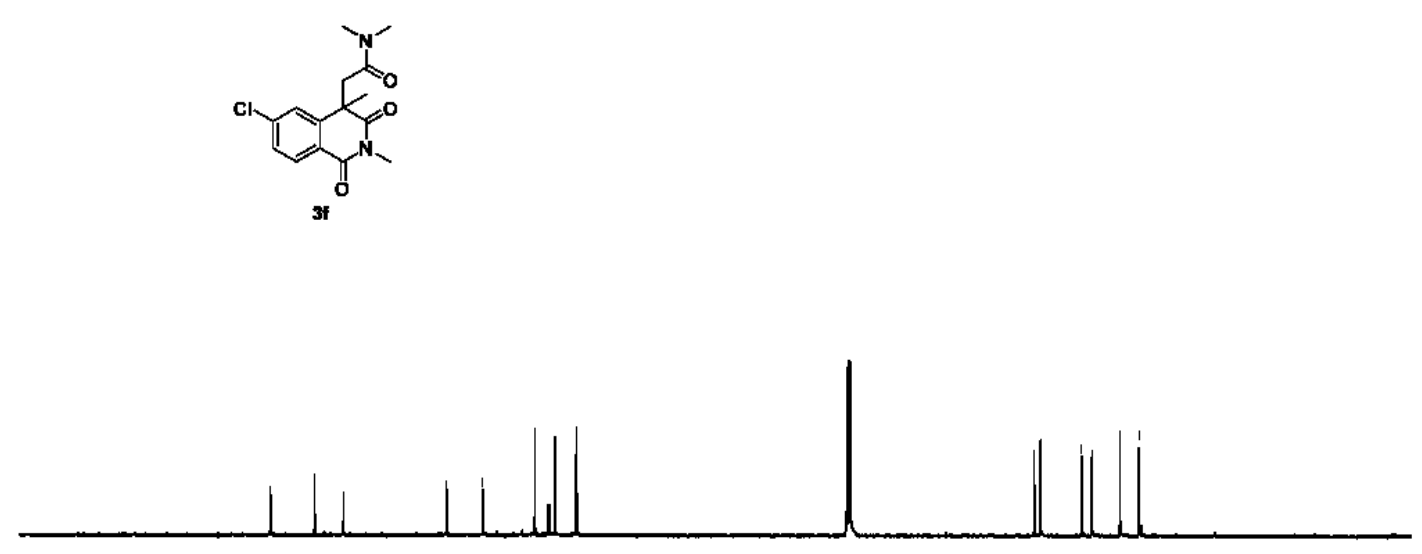

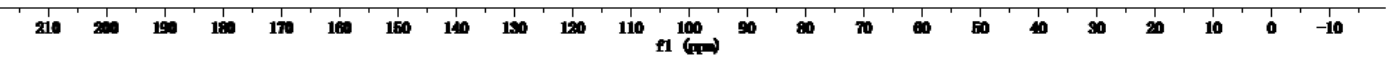



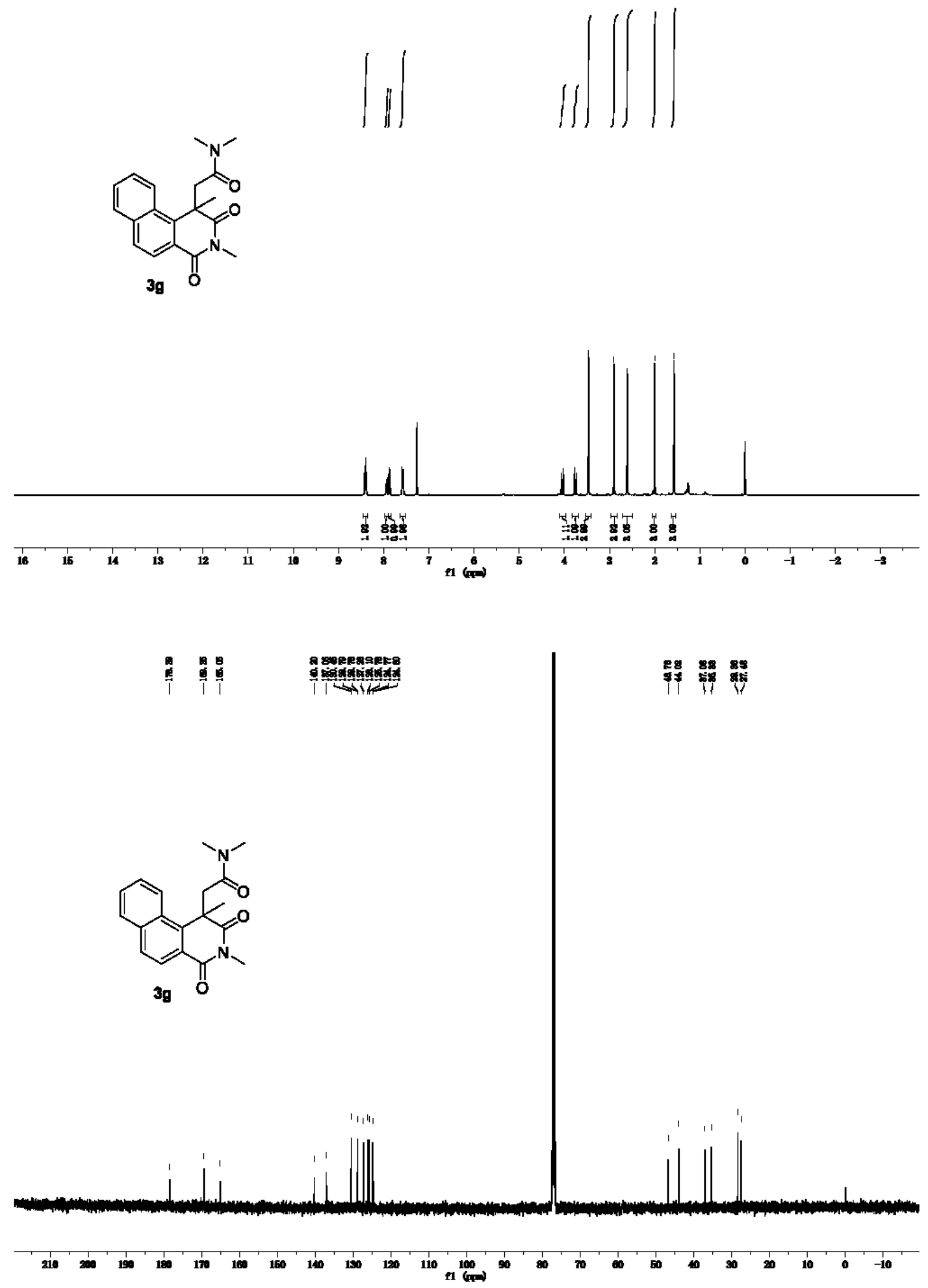

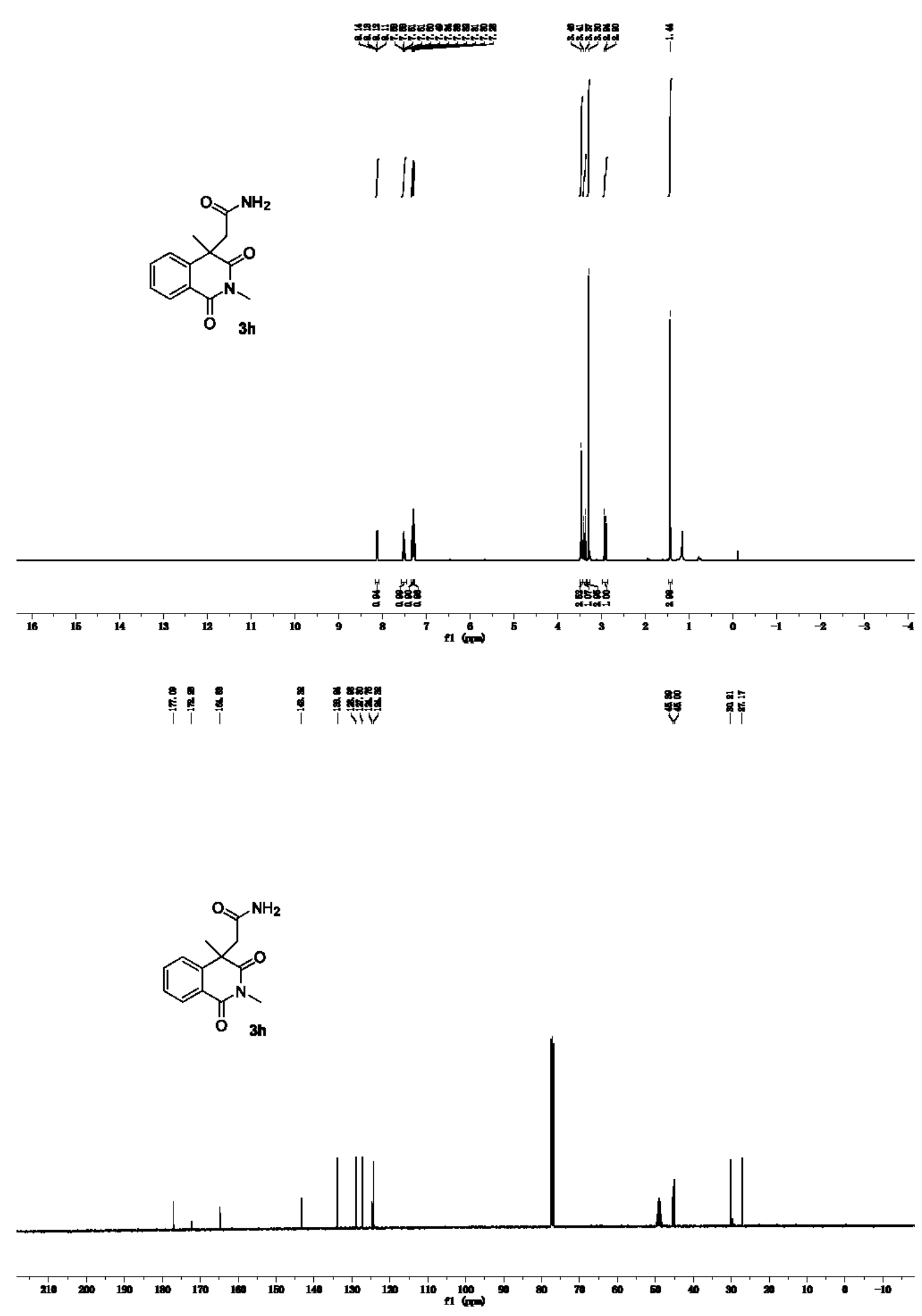


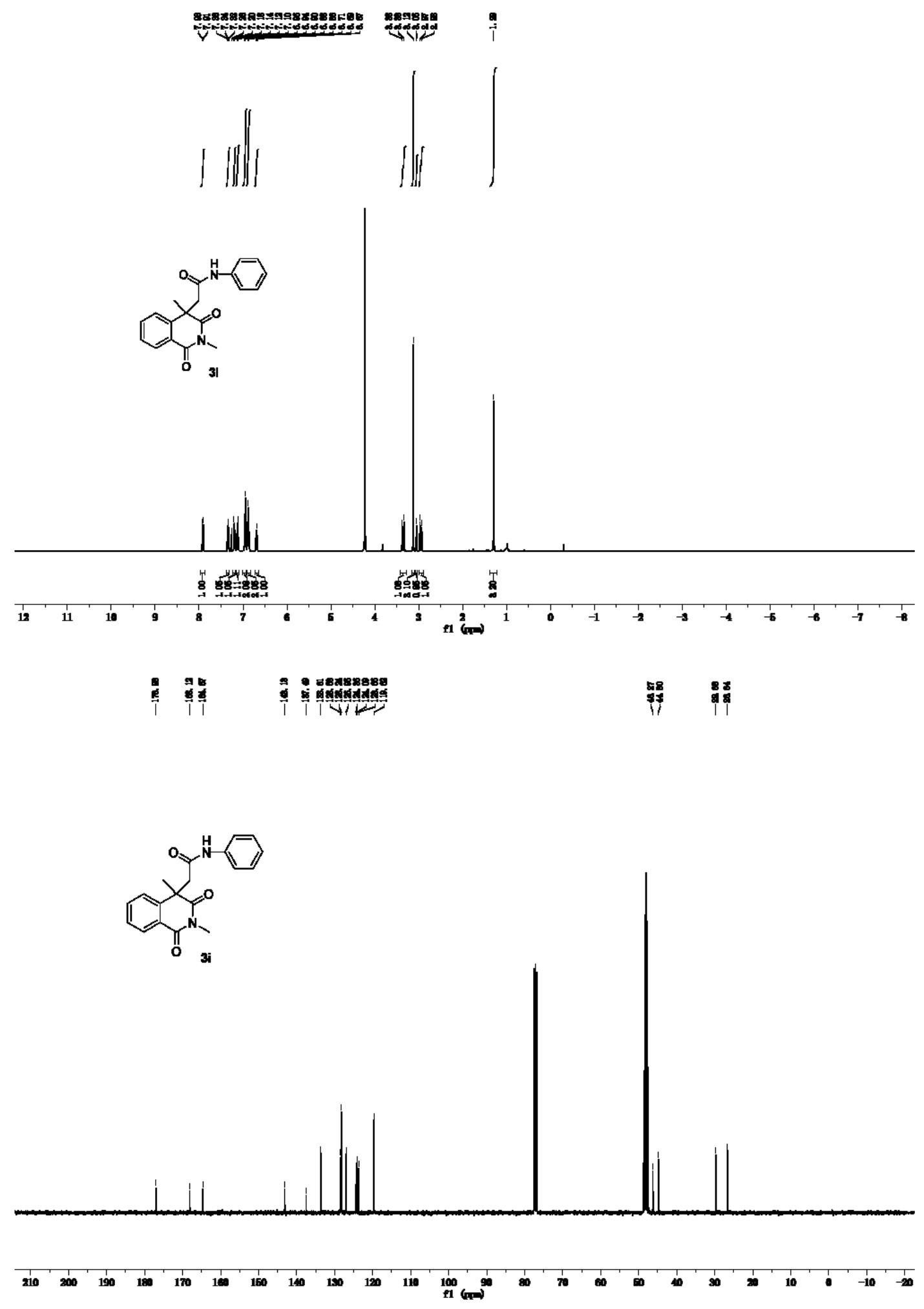




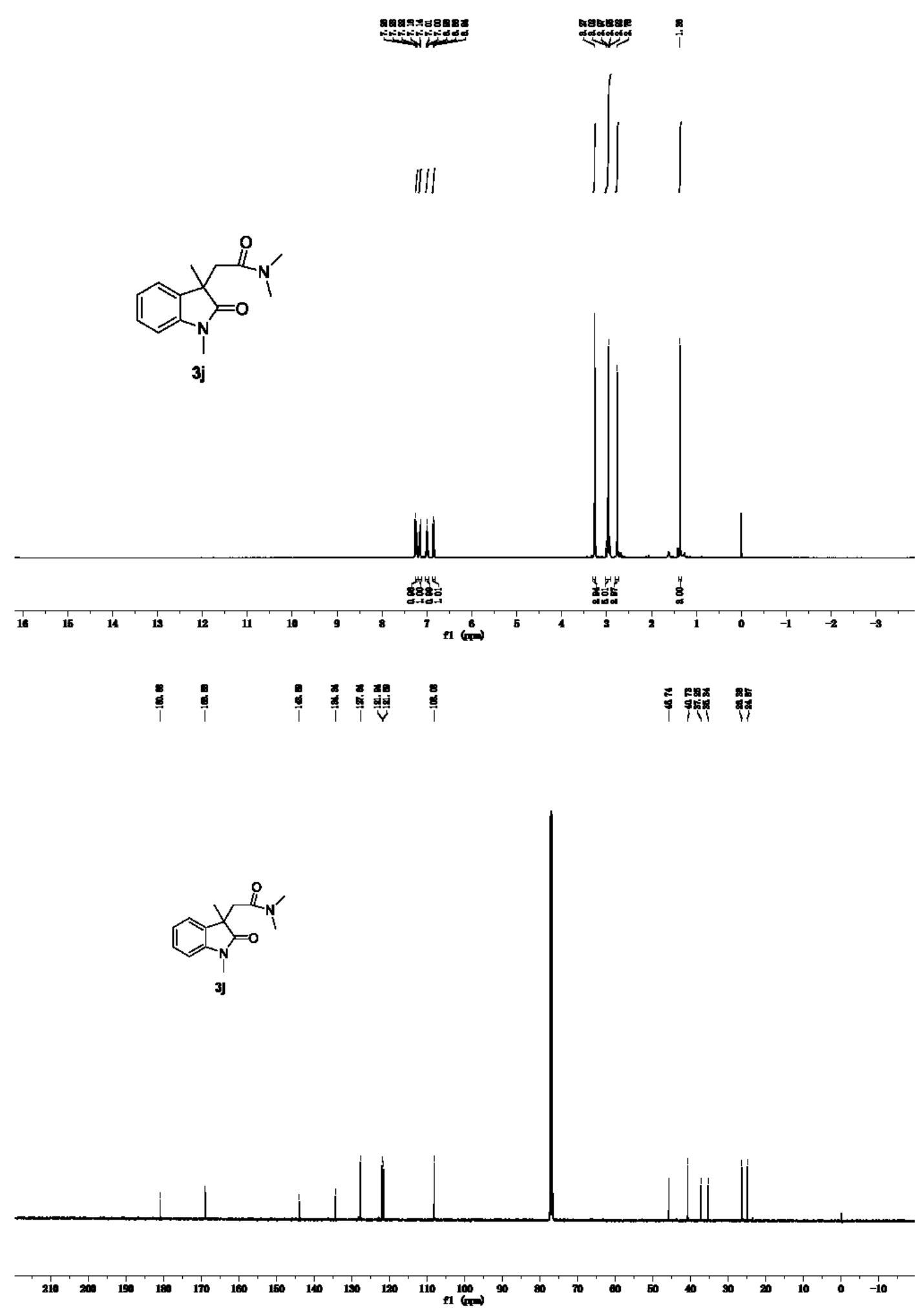


agagsagas

III $\mid$

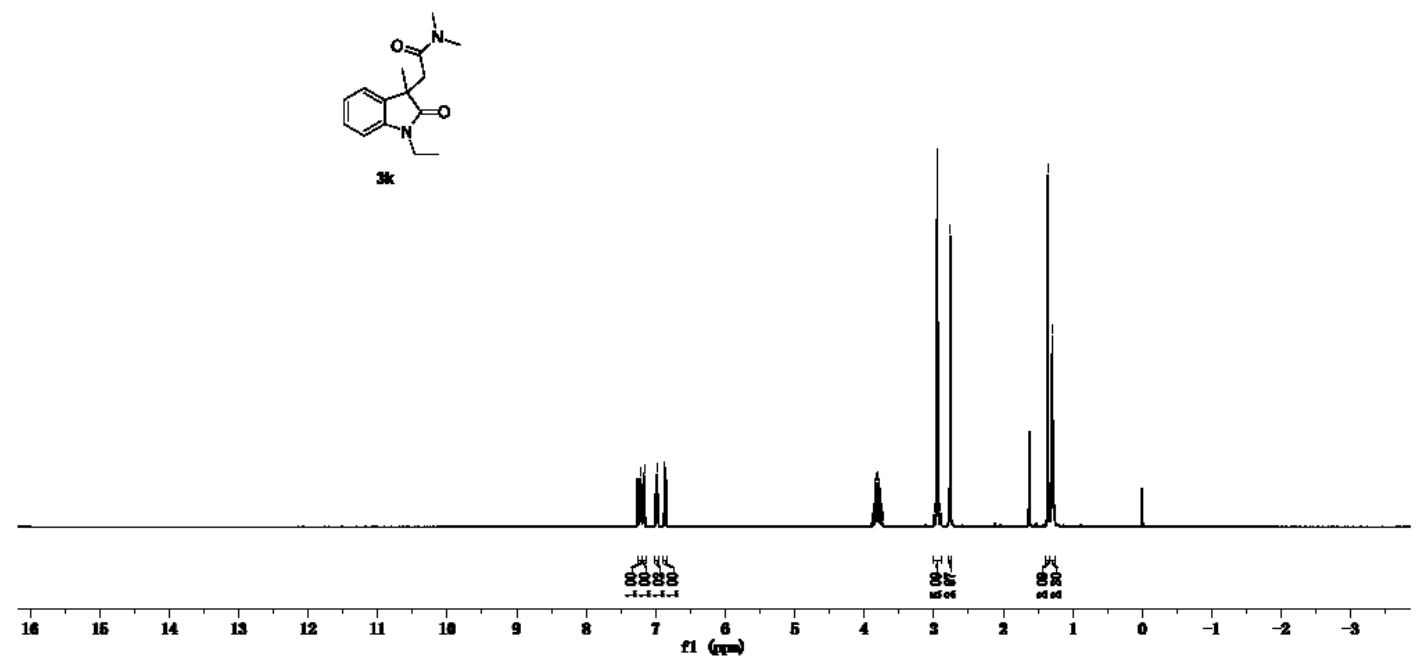

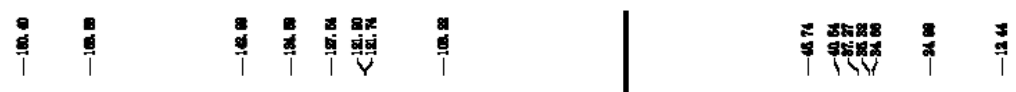

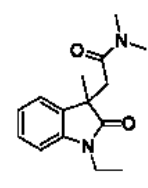

$3 \mathbf{k}$ 
Aasogegse

III $|1|$

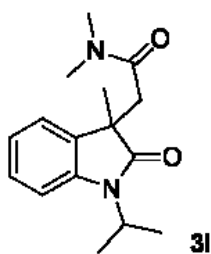

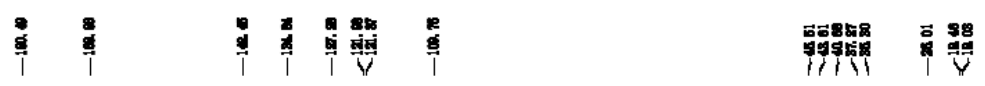<smiles>CC(C)N1C(=O)C(C)(CC(=O)N(C)C)c2ccccc21</smiles>

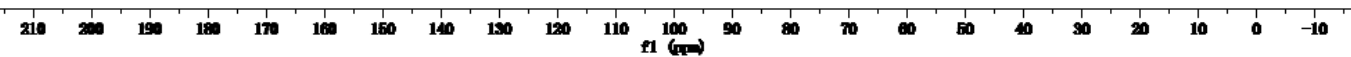



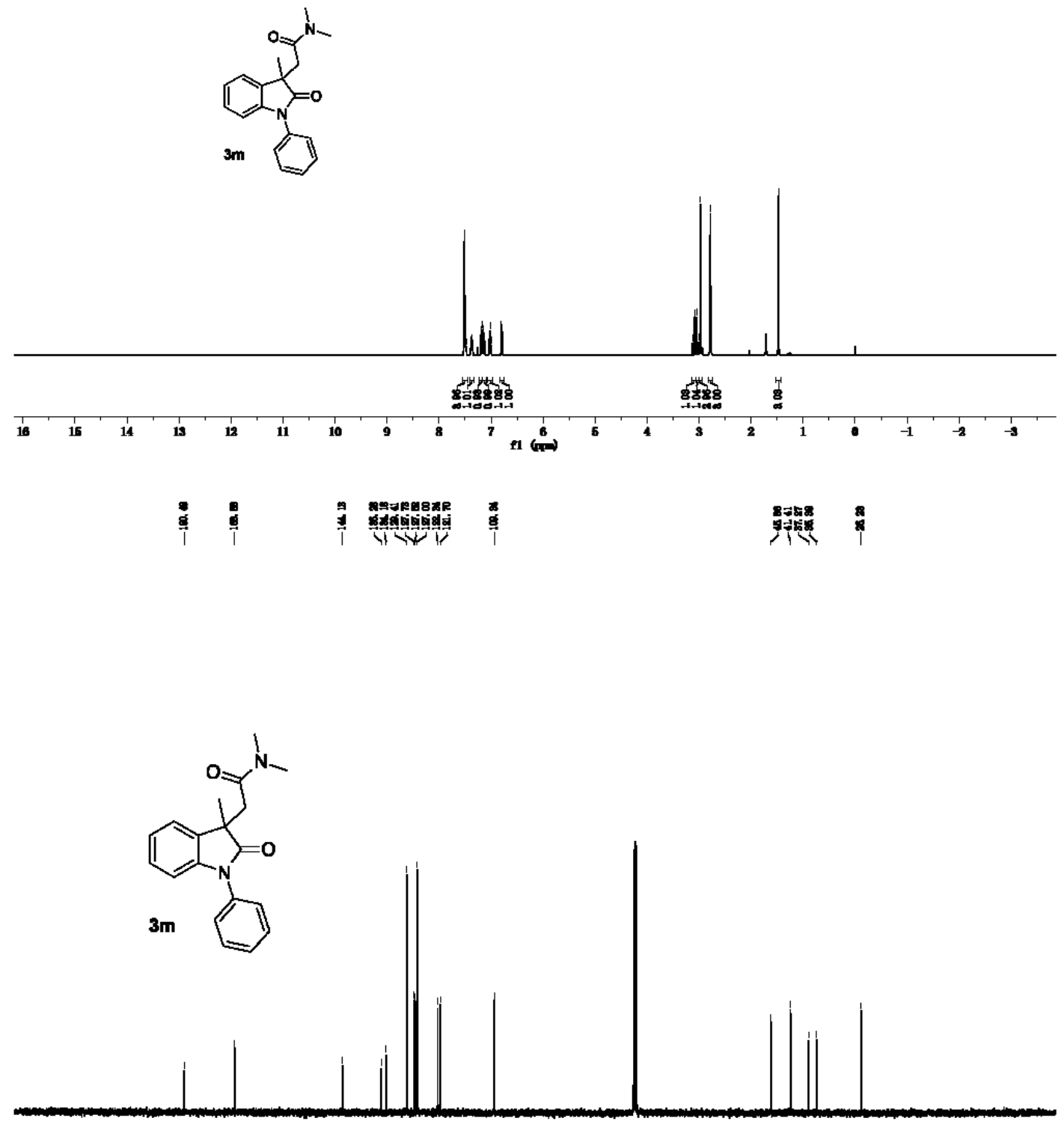


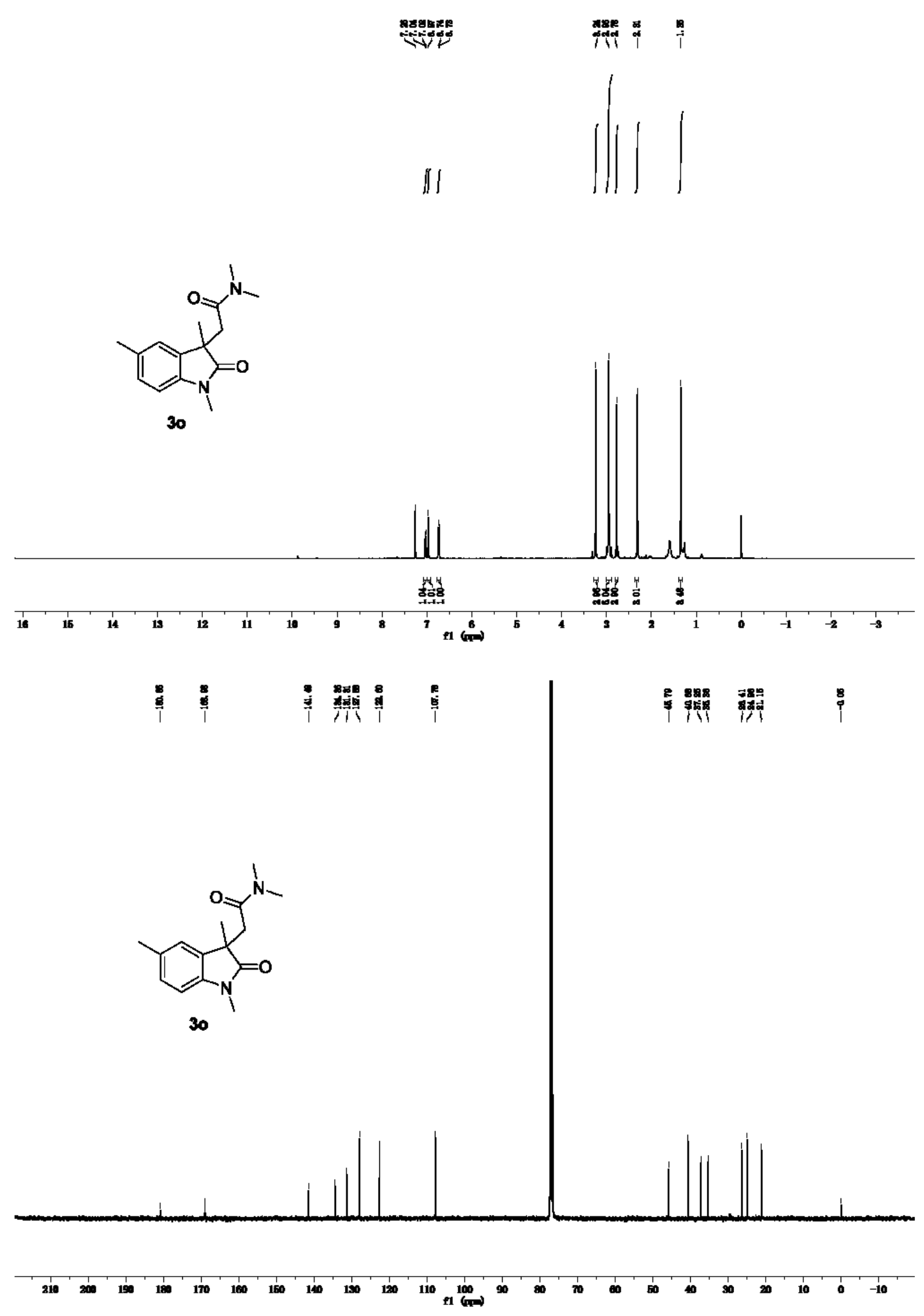




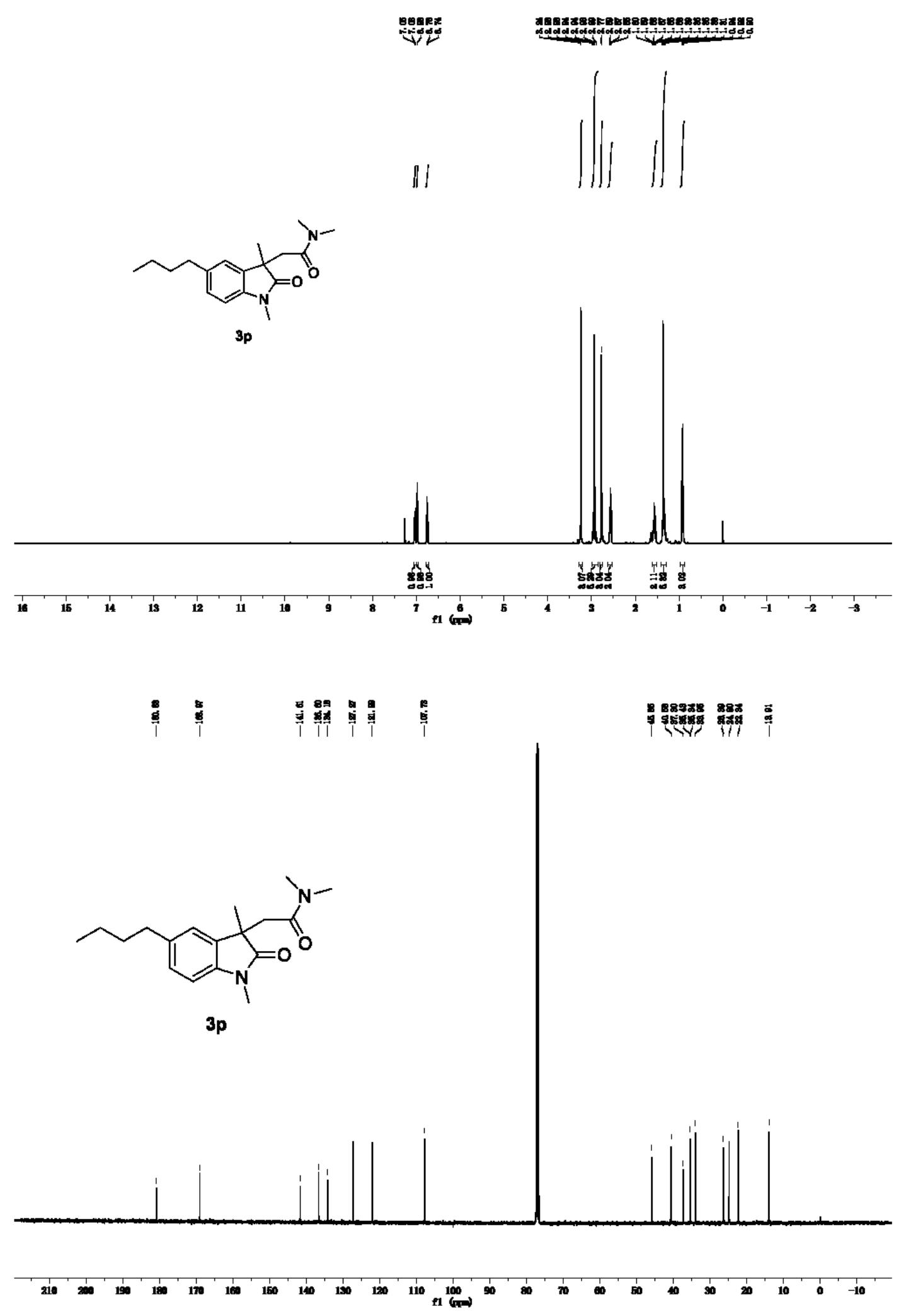




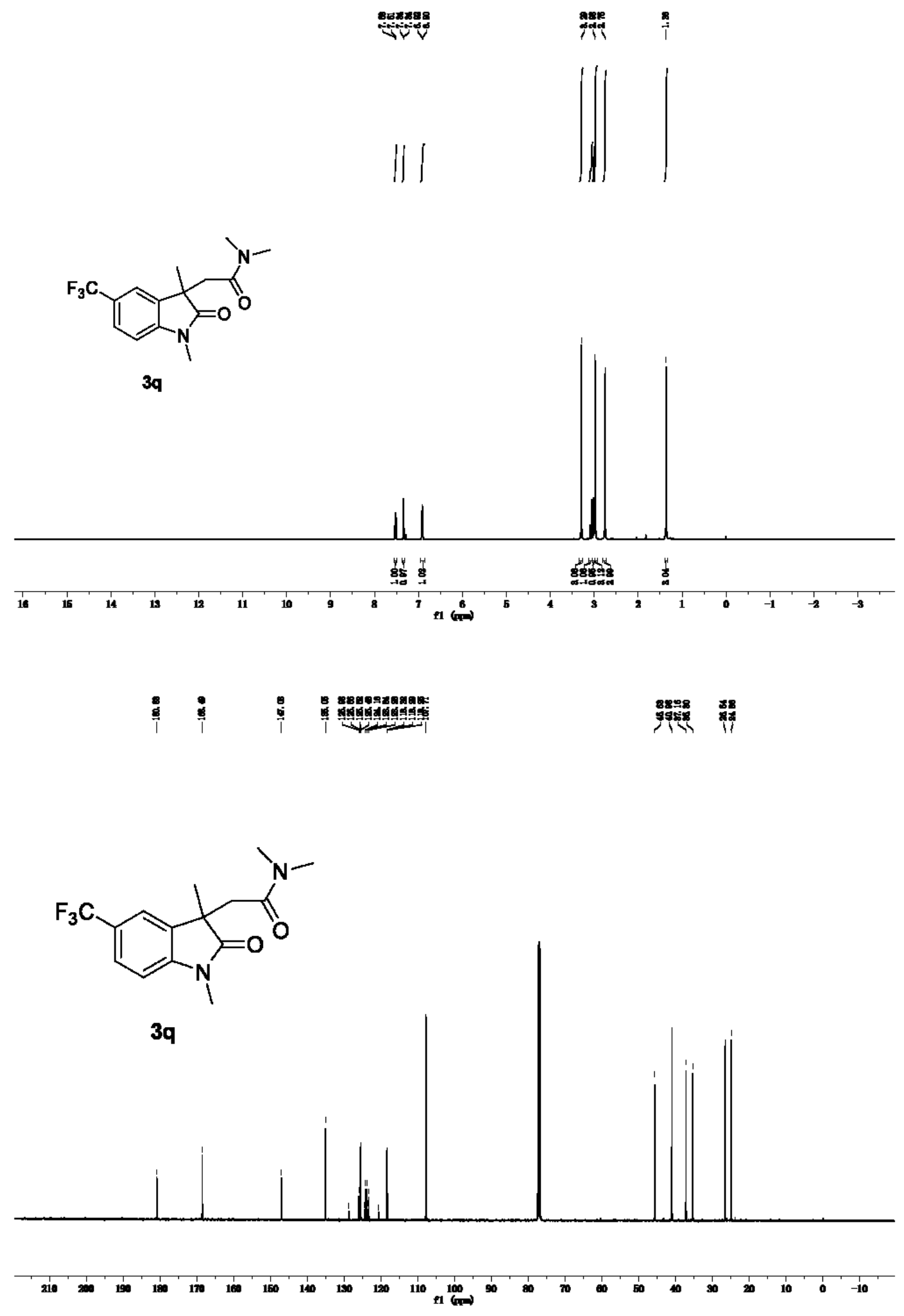



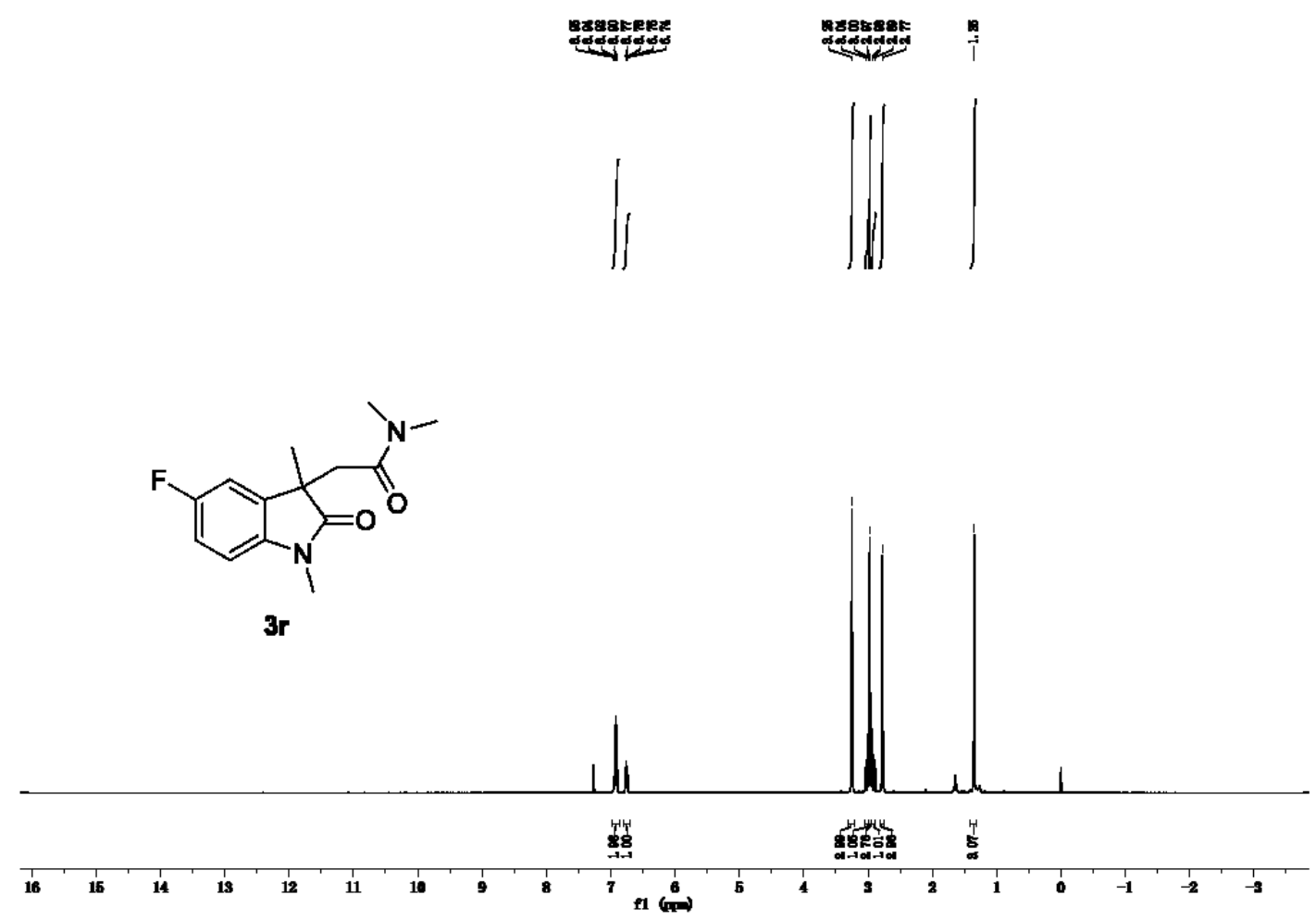

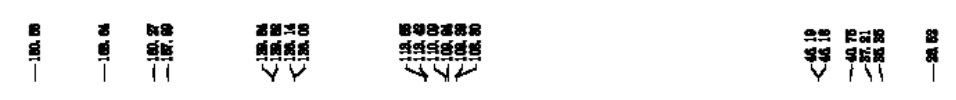

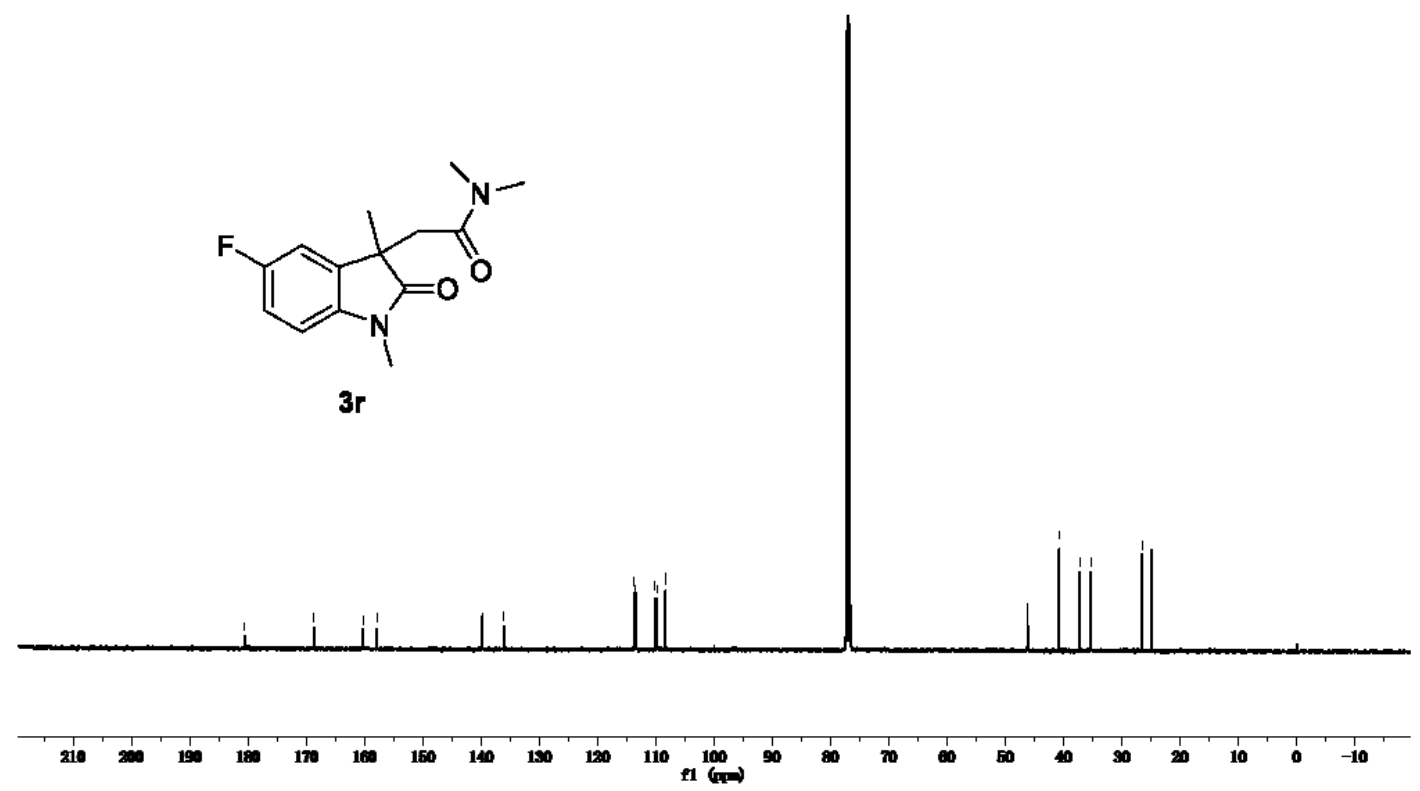




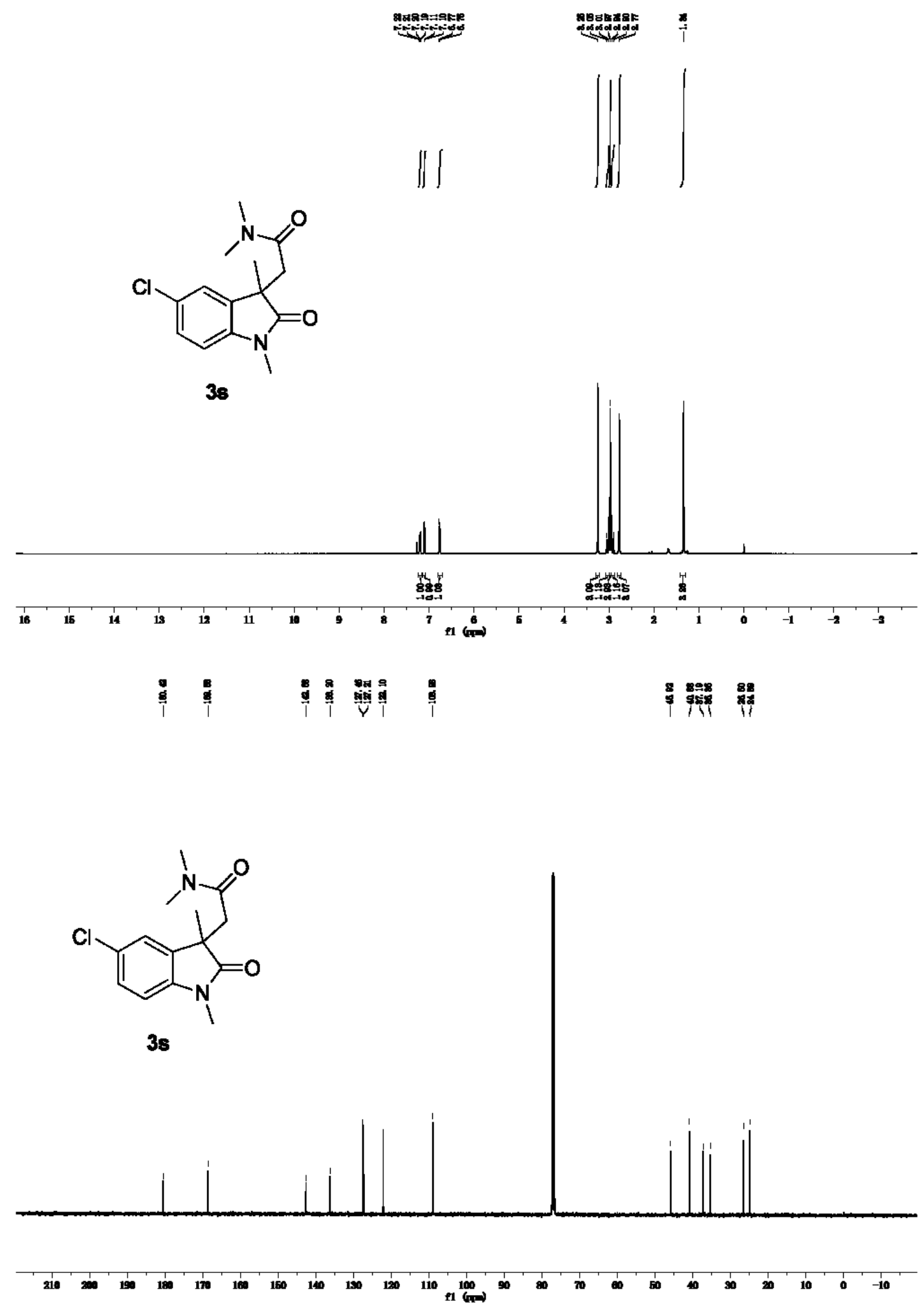



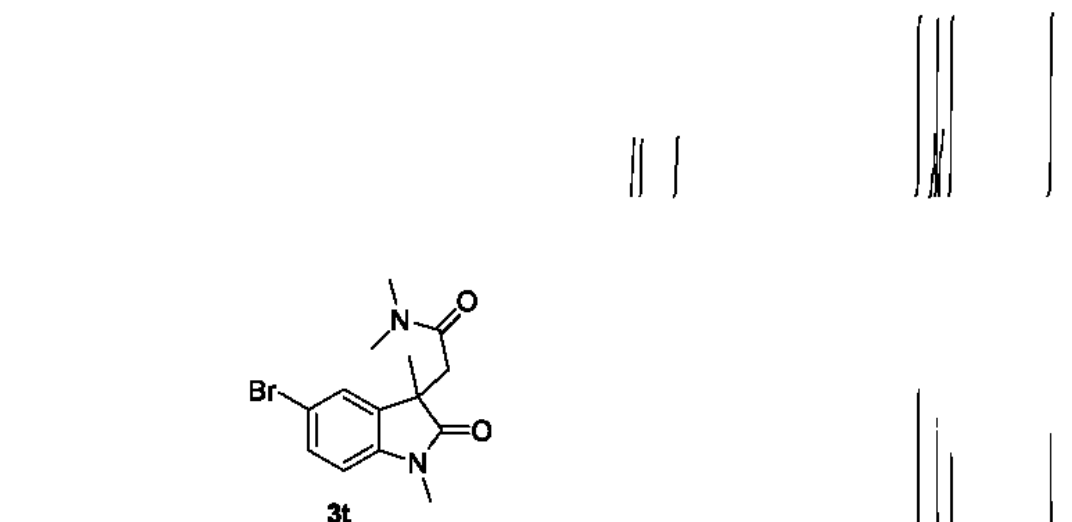

$3 t$

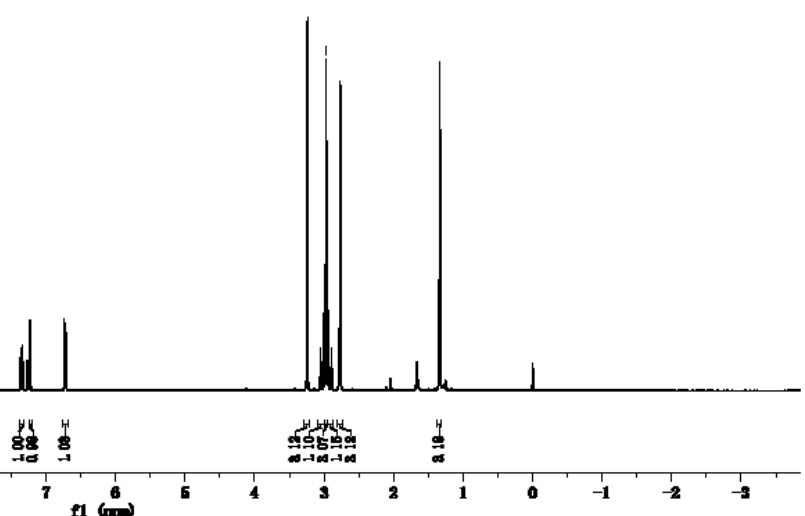

:

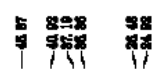<smiles>CN1C(=O)CC2(CC1=O)C(=O)N(C)c1ccc(Br)cc12</smiles>

3t

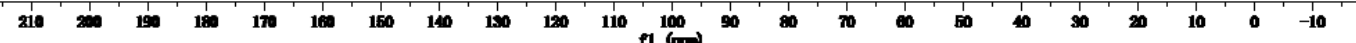




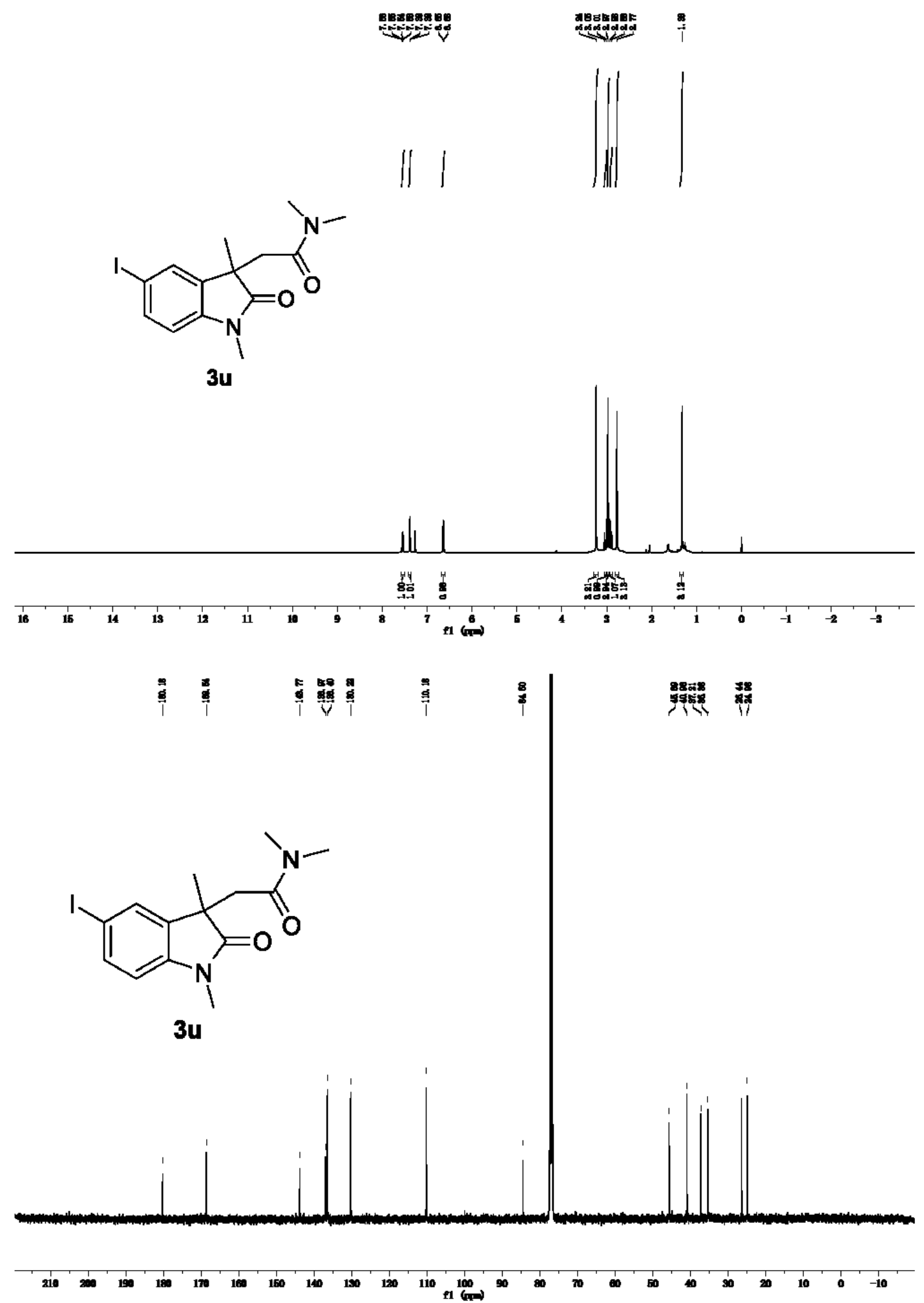


sac

照照

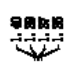

$\iint \quad \int$

$\int||$<smiles>CCOC(=O)c1ccc2c(c1)C(C)(CC(=O)N(C)C)C(=O)N2C</smiles>

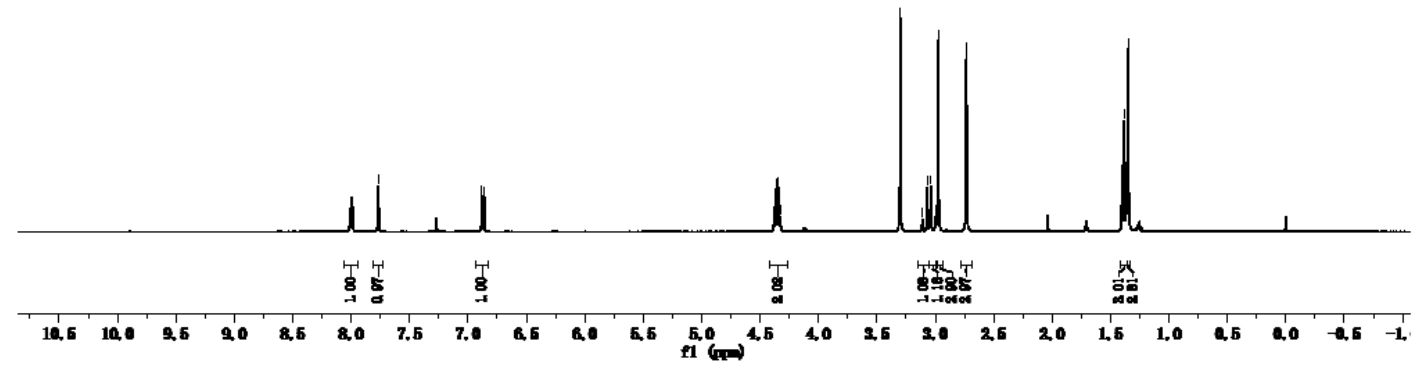

每

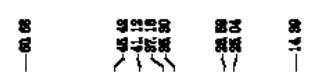

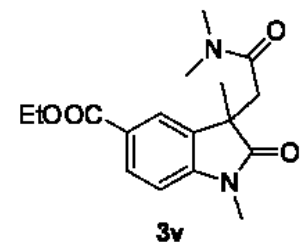

210 210

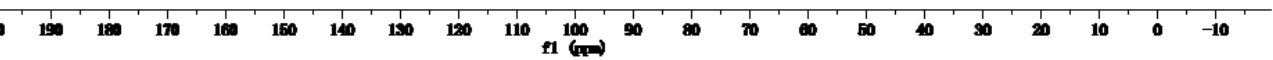



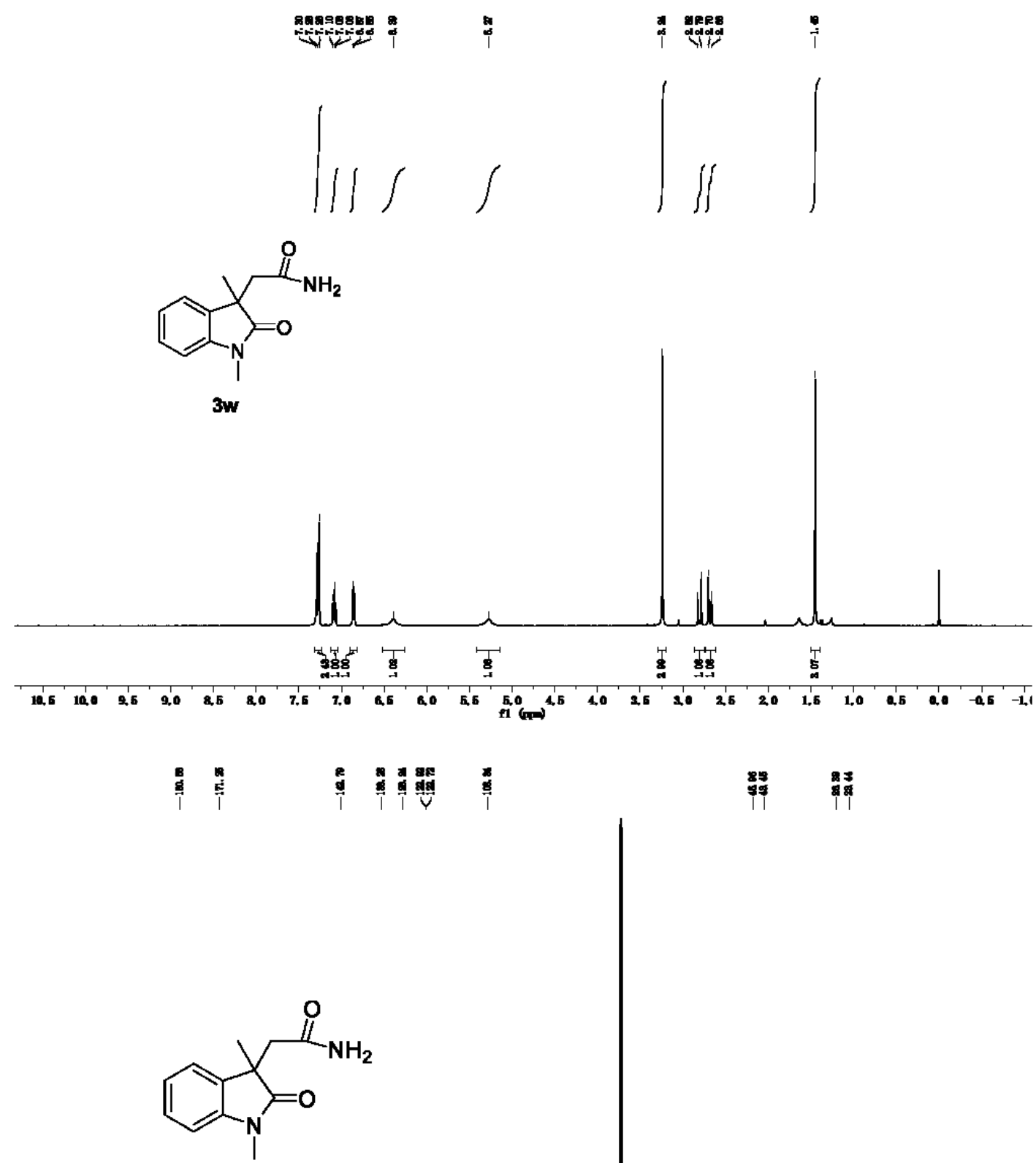

$3 w$

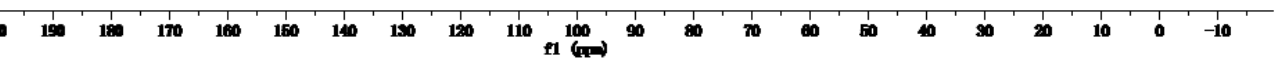




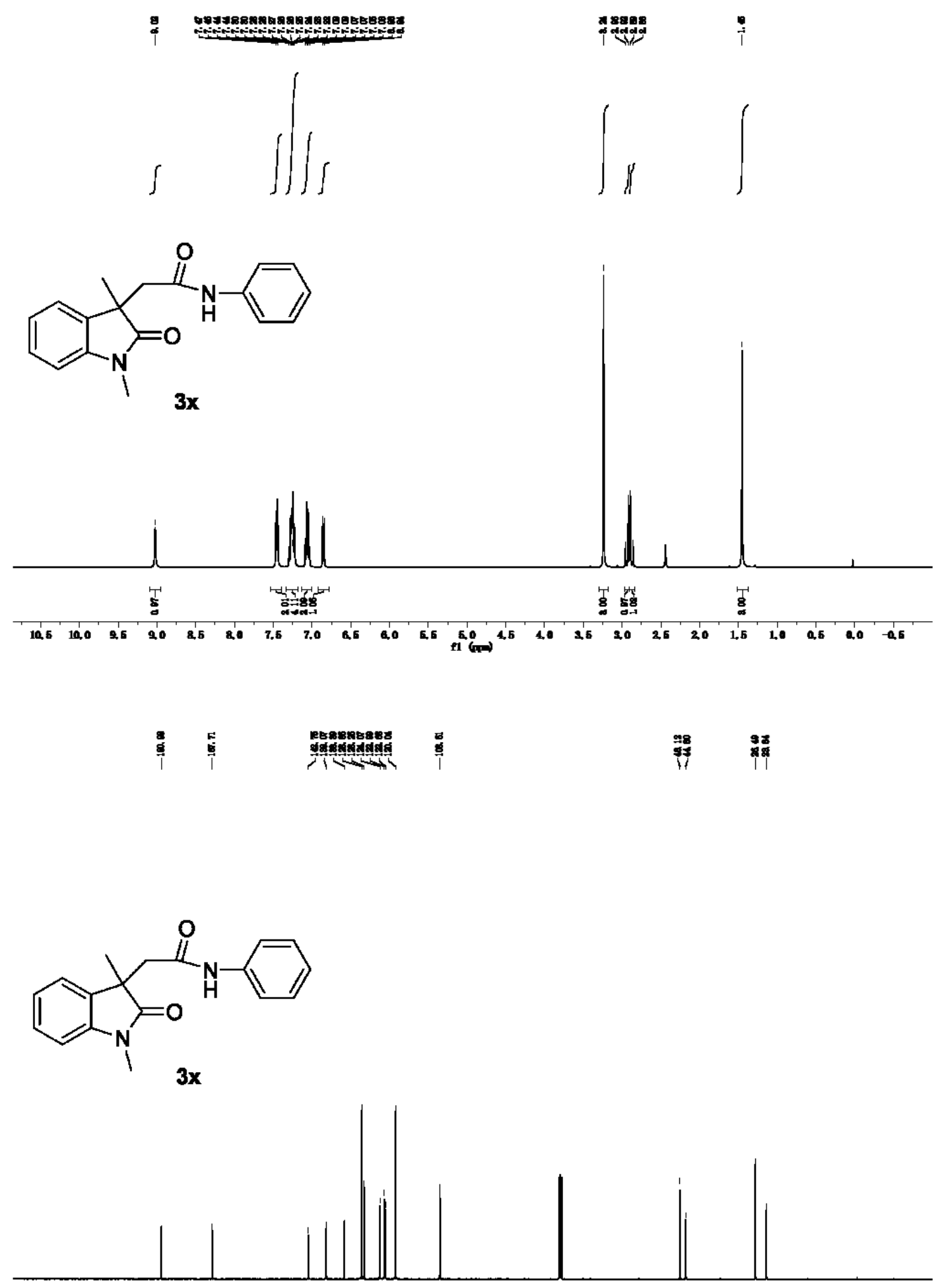

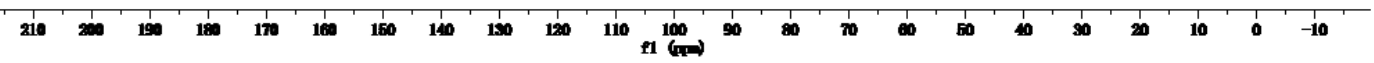

